Aus der Abteilung Allgemeinmedizin

(Prof. Dr. med. M. M. Kochen, MPH, FRCGP)

im Zentrum Innere Medizin

der Medizinischen Fakultät der Universität Göttingen

\title{
Management der tiefen Beinvenenthrombose
}

Veränderungen in Diagnostik und Therapie im Zeitraum von 1990 bis 2003

\author{
INAUGURAL - DISSERTATION \\ zur Erlangung des Doktorgrades \\ der Medizinischen Fakultät \\ der Georg-August-Universität zu Göttingen \\ vorgelegt von \\ Felix K. Schlehahn \\ aus \\ Leipzig
}

Göttingen 2007 
Dek a n:

Prof. Dr. med. C. Frömmel

I. Berichterstatter: Prof. Dr. med. M. M. Kochen, MPH, FRCGP

II. Berichterstatter/in:

III. Berichterstatter/in:

Tag der mündlichen Prüfung: 
1. Einleitung 1

1.1 Einführung 1

1.1.1 Pathogenese 1

$\begin{array}{lll}1.1 .2 & \text { Diagnostik } & 3\end{array}$

$\begin{array}{lll}1.1 .3 & \text { Therapie } & 7\end{array}$

1.1.3.1 Entwicklung der niedermolekularen Heparine (NMH) 7

1.1.3.2 Frühmobilisation $\quad 8$

$\begin{array}{lll}1.2 & \text { Zielsetzung } & 10\end{array}$

2. Patienten und Methoden 12

2.1 Methodische Anlage der Untersuchung 12

$\begin{array}{lll}2.2 & \text { Retrospektiver Teil } & 13\end{array}$

2.2.1 Teilnehmende Patienten und medizinische Einrichtungen 13

2.2.1.1 Phlebologische Schwerpunktpraxis 13

$\begin{array}{ll}\text { 2.2.1.2 Krankenhaus } & 15\end{array}$

$\begin{array}{ll}\text { 2.2.2 Untersuchungsinstrument } & 16\end{array}$

2.3 Prospektiver Teil 17

2.4 Datenanalyse 18

3. Ergebnisse 19

$\begin{array}{ll}3.1 & 19\end{array}$

3.2 Beschreibung der Stichprobe 19

$\begin{array}{lll}3.2 .1 & \text { Praxis } & 19\end{array}$

$\begin{array}{lll}3.2 .2 & \text { Krankenhaus } & 20\end{array}$

3.3 Vorerkrankungen und Risikofaktoren 20

3.4 Thromboseanamnese 21

$\begin{array}{lll}3.4 .1 & \text { Praxis } & 21\end{array}$

3.4.2 Krankenhaus 21

3.5 Thrombophilie 21

3.6 Vormedikation 22

3.7 Symptomatik zum Untersuchungszeitpunkt 22

$\begin{array}{lll}3.8 & \text { Untersuchungsbefunde } & 23\end{array}$

$\begin{array}{lll}3.9 & \text { Diagnostik } & 24\end{array}$

$\begin{array}{lll}3.9 .1 & \text { Praxis } & 24\end{array}$

$\begin{array}{lll}3.9 .2 & \text { Krankenhaus } & 24\end{array}$

$\begin{array}{lll}3.10 & \text { Diagnosestellung } & 25\end{array}$

$\begin{array}{lll}3.10 .1 & \text { Praxis } & 25\end{array}$

3.10.2 Krankenhaus 26

3.11 Therapie 28

3.11.1 Praxis 28

$\begin{array}{ll}3.11 .2 \text { Krankenhaus } & 29\end{array}$

$\begin{array}{lll}3.12 & \text { Entlassungsbrief } & 30\end{array}$

3.13 Prospektive Untersuchung 31

4. Diskussion 36

$\begin{array}{lll}4.1 & \text { Körperliche Symptome } & 37\end{array}$

4.2 Farbduplexsonographie versus Phlebographie 38

4.3 Der Stellenwert der D-Dimer-Testung 40

4.4 Niedermolekulare Heparine versus unfraktionierte Heparine 42

4.5 Ambulante Behandlung einer tiefen Beinvenenthrombose 44

4.6 Zunahme der tVT-Verdachtsfälle 46

5. Zusammenfassung 49

6. Schlussfolgerungen 52

7. Literaturverzeichnis 54 
8. Anhang

63

8.1 Retrospektiver Erfassungsbogen

8.2 Prospektiver Erfassungsbogen

63

65 


\section{Einleitung}

\section{$1.1 \quad$ Einführung}

\subsubsection{Pathogenese}

Bereits im 19. Jahrhundert wurden die wesentlichen Entstehungsmechanismen der tiefen Beinvenenthrombose (tVT) durch den deutschen Pathologen Rudolf Virchow erforscht. Die "Virchow`sche Trias“, die auch in der modernen Medizin nicht an Bedeutung verloren hat, beschreibt die auslösenden Faktoren für die Thromboseentwicklung (Beighton 1968).

- Pathologische Veränderungen der Gefäßwand:

Veränderungen beziehungsweise Beschädigungen der Gefäßwand, zum Beispiel hervorgerufen durch Toxine oder Traumen, können zu einer Umwandlung der Gefäßoberfläche im Sinne eines Verlustes der antithrombogenen Eigenschaften führen.

- Pathologische Veränderungen der Blutzusammensetzung (Thrombophilie):

Veränderungen des Gefäßinhaltes können Thrombosen bewirken. Dem können eine gesteigerte Gerinnbarkeit des Blutes, eine nicht ausreichende Fibrinolyse oder eine ungenügende Inhibitorfunktion zugrunde liegen.

- Pathologisch veränderter Blutfluss (Stase):

Verändert sich die Blutströmung zum Beispiel während einer bestehenden Immobilisation oder auf Grund von Hindernissen im Gefäßsystem können Thrombosen die Folge sein.

Der besondere Stellenwert der tiefen Beinvenenthrombose beruht zum einen auf der potentiell letalen Komplikation einer Lungenembolie, zum anderen auf den chronischen Folgezuständen, die aus diesem Krankheitsbild hervorgehen können. Dabei tritt die Lungenembolie mit einer Häufigkeit von $10-20 \%$ bei den betroffenen Patienten auf (Kraaijenhagen et al. 1997). Die aus der Erkrankung an einer tVT langfristig entstehenden Begleitkomplikationen werden unter dem Begriff des postthrombotischen Syndroms zusammengefasst. Es tritt bei ca. 50\% der Patienten 5-10 Jahre nach dem Ereignis auf (Brandjes et al. 1997). Hierbei führt die Zerstörung der Venenklappen zu einer Insuffizienz im venösen Schenkel und im weiteren Verlauf zu Ödemen und Ulzerationen (Müller-Lisse et al. 1998). Diese Folgeerkrankungen füh- 
ren zu einer erheblichen Einschränkung der Lebensqualität und über die langen Behandlungszeiten zu einem bedeutenden Kostenaufwand (Müller-Lisse et al. 1998).

In Deutschland treten pro Jahr 200.000 tiefe Beinvenenthrombosen auf (Spengel und v. Liebe 1998). Die weltweite Inzidenz liegt bezogen auf 1000 Einwohner bei 1 bis 2 Erkrankungen im Jahr (Kearon 2001).

Zahlreiche Umstände erhöhen das Risiko, eine tVT zu entwickeln. Vor allem die ererbten und erworbenen Thrombophilien sind prädisponierende Faktoren (Martinelli et al. 1998). So wird der prozentuale Anteil der durch Thrombophilien hervorgerufenen Thrombosen mit etwa 50\% angegeben (Seligsohn und Lubetsky 2001). Ab der 6. Dekade stellt das Alter den dominierenden unabhängigen Einflussfaktor einer tVT dar (Heit et al. 2001). Bei jüngeren Patienten ist der Anteil der durch hereditäre Thrombophilien hervorgerufenen Thrombosen größer (van Boven et al. 1999). Klassische Auslöser für eine gesteigerte Thrombosebereitschaft sind eine bestehende Immobilisation, eine vorangegangene Operation und ein bekanntes Malignom (Mateo et al. 1997). Ebenso ist unter der Einnahme von Östrogenpräparaten mit einer gesteigerten Blutgerinnung zu rechnen (Wells et al. 1997).

Die Zunahme der Erkrankungshäufigkeit im unmittelbaren Zusammenhang mit einer längeren Reise bei vorher asymptomatischen Patienten führte zu einer Sensibilisierung der Bevölkerung bezüglich der tiefen Beinvenenthrombose. Bereits 1968 wurde die hohe Inzidenz venöser Thrombosen nach Langstreckenflügen beschrieben (Beighton 1968). Der Begriff der sogenannten „economy-class-syndrom“ wurde geprägt, der im Deutschen auch als „Reisethrombose“ bezeichnet wird. 


\subsubsection{Diagnostik}

\section{Klinische Untersuchung}

Das klinische Bild einer tVT variiert zwischen den beiden Extremen einer Phlegmasia coerulea dolens und völlig inapparenten Verläufen. Erstere geht auf Grund einer Massenvenenthrombose mit starken Schmerzen und Schwellungen einher. Diese Schwankungen in der Symptomatik einer IVT haben zur Verwendung zahlreicher klinischer Untersuchungszeichen und Tests geführt (Tabelle 1), die in die Diagnostik einer IVT einfließen können. Der angegebene Fußsohlendruckschmerz erklärt sich dabei aus der Tatsache, dass sich Thrombosen im Bereich des Unterschenkels häufig aus einer Plantarvenenthrombose entwickeln (Kearon et al. 1998).

Tabelle 1: Klinische Zeichen einer tiefen Beinvenenthrombose (Fischer 2004, S. 247)

\begin{tabular}{ll}
\hline Eigenname & Beschreibung \\
\hline Payr-Zeichen & $\begin{array}{l}\text { Fußsohlendruckschmerz im Bereich der medialen Plantar- } \\
\text { muskulatur; entspricht dem Schmerz, den der Patient spon- } \\
\text { tan beim Auftreten angibt }\end{array}$ \\
$\begin{array}{l}\text { Homan-Zeichen } \\
\text { Bisgaard-Zeichen }\end{array}$ & $\begin{array}{l}\text { Wadenschmerz bei Dorsalflexion des Fußes } \\
\text { Pratt-Zeichen }\end{array}$ \\
Sigg-Zeichen & $\begin{array}{l}\text { Druckschmerz in der Kniekehle } \\
\text { Schmerzen in der Kniekehle bei Überstreckung }\end{array}$ \\
Ducuing-Zeichen & Schmerz bei manueller Kompression der Wadenmuskulatur \\
Lowenberg-Test & $\begin{array}{l}\text { Wadendruckschmerz bei Druck >100 mmHg mittels Blut- } \\
\text { druckmanschette, nur aussagekräftig bei Seitendifferenz }\end{array}$ \\
Meyer-Druckpunkte & Druckschmerz medial der Tibiakante \\
\hline
\end{tabular}

Tabelle 2 zeigt die eher mäßigen Testgütekriterien klinischer Thrombosezeichen. Die Datenbasis hierzu beruht auf einer Reihe von Studien mit einer sehr geringen Fallzahl berücksichtigter Patienten (meist weniger als 100). Keines dieser Zeichen erlaubt eine sichere Ausschlussdiagnose (Ebell 2001, Kearon et al. 1998). Eine umfangreichere Studie von Aschwanden et al. (1999) mit 343 Patienten bestätigte die nur geringe Sensitivität (67\%-84\%) und Spezifität (51\%-56\%) der klinischen Untersuchung. 
Die mangelnde Aussagekraft einzelner Zeichen führte zur Entwicklung zusammenfassender Scores. Die größte Bedeutung erlangte in den letzten Jahren die Einteilung nach Wells et al. (2003). Orientierend am Schweregrad der vorhandenen Klinik wird hierbei anhand einer Punkteskala die klinische Wahrscheinlichkeit für das Bestehen einer tiefen Beinvenenthrombose errechnet. Jedoch konnte auch bei diesem Score eine Restunsicherheit von 2-4\% belegt werden. Aus der Sicht der primärärztlichen Versorgung kommt erschwerend hinzu, dass diese Studien zumeist in Einrichtungen der Sekundär- oder Tertiärversorgung durchgeführt wurden. Die niedrige Prävalenz der tVT im primärärztlichen Bereich führt zu noch niedrigeren Testgütekriterien und damit zu einer noch schlechteren Aussagekraft klinischer Zeichen (Fischer et al. 2004). Dies geht konform mit einer aktuellen Studie von Oudega et al. (2005 a), bei der 1295 hausärztliche Patienten (mit dem Score von Wells) untersucht wurden. Allein Patientenanamnese und körperliche Untersuchung erlauben auch mittels des Einsatzes des Wells-Scores keine ausreichend sichere Aussage bezüglich der Erkrankungswahrscheinlichkeit (Oudega et al. 2005 a). Für die Patientengruppe mit niedrigem Erkrankungsrisiko ermittelten die Autoren einen Anteil von $12 \%$, bei denen eine tiefe Beinvenenthrombose vorlag.

Tabelle 2: Testgütekriterien körperlicher Untersuchungsbefunde (Ebell 2001, S.168)

\begin{tabular}{lccc} 
& gesamt & Sensitivität (\%) & Spezifität (\%) \\
\hline Immobilisation & 593 & 24 & 90 \\
Venöse Gefäßerweiterung & 15 & 25 & 89 \\
Schwellung & 102 & 84 & 44 \\
Temperaturdifferenz & 50 & 72 & 48 \\
Ödeme & 50 & 97 & 33 \\
Schmerzen & 50 & 86 & 19 \\
Lokale Empfindlichkeit & 102 & 76 & 11 \\
Erythem & 102 & 24 & 62 \\
\hline
\end{tabular}

Sowohl aus gesundheitsökonomischer Sicht als auch aus der Perspektive der Patienten wäre es jedoch wünschenswert, wenn entweder allein mittels der klinisch körperlichen Untersuchung oder in Verbindung mit einem ambulant durchführbaren (und kostengünstigen) Laborverfahren eine sichere Diagnose gestellt werden könnte. Unter diesen Voraussetzungen ergäbe sich auf Grund einer möglichen „Filterfunktion“ des Hausarztes eine Reduktion der sonst nötigen apparativen und kostenintensiven 
Diagnostik. Neben Einsparungen für das Gesundheitssystem würde dies auch Vorteile für den Patienten mit sich bringen. Die Risiken invasiverer Verfahren ließen sich somit für viele Patienten vermeiden. Bislang ist jedoch unklar, inwieweit Hausärzte bereits jetzt eine Filterfunktion wahrnehmen. Es liegen keine Daten zu Charakteristika hausärztlich mit tVT-Verdacht zum Spezialisten überwiesener Patienten vor.

\section{Phlebographie}

Die Phlebographie stellte laut einiger Autoren Ende der neunziger Jahre noch den Goldstandard in der Diagnostik der tiefen Beinvenenthrombose dar (Ginsberg et al. 1997, Herrmann et al. 1998). Aktuell wird das Verfahren auf Grund seiner Invasivität in den Hintergrund gedrängt (Cogo et al. 1998). Im Vergleich sind bei sonographischer Untersuchung Komplikationen, wie allergische Reaktionen auf das Kontrastmittel, Nierenschädigungen und Belastungen des Patienten durch Röntgenstrahlen ausgeschlossen. Die Phlebographie zeigt zudem eine schwankende Aussagekraft, da falsch positive und negative Befunde möglich sind (Rudofsky und Michler 2002). So konnte das Verfahren in 3-5\% der Fälle die durch eine pathologische Untersuchung festgestellte Thrombose nicht nachweisen (Rudofsky und Michler 2002). Ebenso ist die Darstellbarkeit der im Unterschenkel gelegenen V.-tibialis-anteriorGruppe häufig nicht möglich (Bjorgell et al. 2000).

\section{Sonographie}

Im Laufe der letzten 15 Jahre wurden mehrere Verfahren entwickelt, die zu einer verbesserten Diagnosestellung führen sollten. Dabei nimmt vor allem die Farbduplexsonographie einen besonderen Stellenwert ein. Sie stellt eine Kombination aus der Dopplersonographie und der Echtzeitsonographie dar. Im Vergleich mit anderen Verfahren erhält der Untersucher zusätzliche Informationen über den Blutfluss (Rudofsky und Michler 2002). Kaliberschwache Muskelvenen im Unterschenkel, umspülte Thromben sowie Teilkanalisierungen können leichter diagnostiziert werden (Dietrich und Bauersachs 2002). In zahlreichen Studien wurde die Sensitivität und Spezifität dieses Verfahrens geprüft. Die ermittelte Sensitivität lag zwischen 0,89 (Ebell 2001) und 0,62-0,97 (Dietrich und Bauersachs 2002). Autoren haben die Aussagekraft der Duplexsonographie mit der der Phlebographie verglichen. Hierbei kamen sie zu dem Ergebnis, dass mittels der Ultraschalluntersuchung in einem hohen Anteil der Fälle sicher und effektiv eine Diagnosestellung möglich ist (Dietrich und Bauersachs 2002). Das Vorgehen bei einem Patienten mit Verdacht auf eine tVT 
sollte demnach zunächst eine Duplexsonographie beinhalten (Kearon et al. 2001). Die geringere Komplikationsrate und die deutliche Kostenreduktion zeichnen diese Untersuchungsmethode zusätzlich aus (Rudofsky und Michler 2002).

\section{D-Dimer}

Dieser in den 70iger Jahren erstmals beschriebene Marker kann zur Diagnostik tiefer Beinvenenthrombosen eingesetzt werden. Dabei handelt es sich um Fibrinspaltprodukte, die im Rahmen einer gesteigerten Gerinnung oder bei im Abbau befindlichen Blutgerinnseln vermehrt auftreten (Grohmann 1999). Die D-Dimer-Konzentration korreliert orientierend mit der Thrombusgröße und -oberfläche (Kelly et al. 2002). Da 2$3 \%$ des Plasma-Fibrinogens permanent zu Fibrin umgewandelt werden, liegt der Referenzwert, ab dem ein relevanter tVT-Verdacht besteht, bei $0,5 \mu \mathrm{g} / \mathrm{ml}$ (Kelly et al. 2002). Im Plasma beträgt die Halbwertszeit der D-Dimere ca. 8 Stunden (Bradley et al. 2000).

Die Sensitivität des Verfahrens schwankt laut Literaturangaben zwischen 85 und 98\% (Aschwanden et al. 1999, Perrier und Bounameaux 2001). Somit lässt sich eine tVT durch Bestimmung der D-Dimere mit hoher Sensitivität erfassen (Legnani et al. 1999). Der niedrige Wert der Spezifität stellt den Nachteil der D-Dimer-Bestimmung dar. Ursächlich ist die Erhöhung der D-Dimer-Konzentration als Folge anderer Erkrankungen, die mit einer verstärkten Aktivität des Gerinnungssystems einhergehen (Le Gal et al. 2006). Hierzu zählen konsumierende Erkrankungen, Infektionen, sowie postoperative und posttraumatische Zustände (Kozman et al. 1997). Eine hohe Rate an falsch positiven Befunden ist die Folge. Mehrere Studien, die die Spezifität unterschiedlicher Testverfahren zur D-Dimer-Bestimmung untersuchten, zeigten für den Elisa-Test Werte von 32-46\%, für den Simpli-Red-Test Werte von 54-75\% (Kelly et al. 2002). Die Kontrolle der D-Dimer-Werte erlaubt in Verbindung mit anderen Untersuchungsverfahren eine Diagnosestellung (Crippa et al. 1997). Die Kombination aus niedriger klinischer Vortest-Wahrscheinlichkeit und niedrigem D-Dimer-Test (Werte $<500 \mathrm{E} / \mathrm{ml}$ ) führt in ca. 98\% der Fälle zum Ausschluss einer tiefen Beinvenenthrombose (Bernardi et al. 1998, Perrier et al. 1999, Kearon et al. 2001, Wells et al. 2003). Somit bietet dieses Verfahren den Ansatz für eine sichere Ausschlussdiagnostik in der Hausarztpraxis. Gegenüber den anderen Verfahren zeichnet sich die Methode durch die Schnelligkeit der Bestimmung, die niedrige Rate an möglichen Komplikationen und den geringen Kostenaufwand aus (Aschwanden et al. 1999). Inwieweit die 
D-Dimer-Bestimmung in den primärärztlichen Bereich umgesetzt werden kann, ist jedoch nach wie vor unklar.

\subsubsection{Therapie}

Die Entwicklung neuer therapeutischer Möglichkeiten führte in den letzten zehn Jahren zu zahlreichen Veränderungen in der Behandlung der tiefen Beinvenenthrombose. Vor allem die Immobilisation des Patienten und die über die Thromboplastinzeit gesteuerte 7- bis 14- tägige Gabe eines Heparins werden heute als unnötig erachtet. Hierfür sind zwei Fortschritte grundlegend gewesen:

a) Die Entwicklung niedermolekularer Heparine, die in fixen gewichtsadaptierten Dosen subkutan injiziert werden können. Hierdurch entfallen die häufig nur unter stationären Bedingungen durchführbaren engmaschigen Laborkontrollen.

b) Die Beobachtung, dass die frühe Mobilisation des Patienten gegenüber der strengen Bettruhe als mindestens gleichwertig anzusehen ist.

\subsubsection{Entwicklung der niedermolekularen Heparine (NMH)}

Mit der Entwicklung niedermolekularer Heparine entstanden neue Möglichkeiten der Prophylaxe venöser Thrombembolien. Diese durch Fragmentierung aus unfraktioniertem Heparin entwickelten Formen haben ein Molekulargewicht von 3200-6500 Dalton (Fareed et al. 1988). Die pharmazeutischen Unterschiede zwischen den beiden Medikamentengruppen bewirken eine bessere Bioverfügbarkeit der niedermolekularen Heparine. Bei subkutaner Applikation weisen sie eine längere Eliminationshalbwertszeit auf (Diehm et al. 2002). Da die NMH lediglich einmal beziehungsweise zweimal am Tag injiziert werden müssen, ist eine ambulante Therapieführung möglich (Tovey und Wyatt 2003). Sie zeigen eine geringere Inzidenz heparininduzierter Thrombozytopenien (HIT). Das Risiko eine HIT unter Verwendung niedermolekularer Heparine zu entwickeln wird bei internistischen Indikationen mit 0,2\% pro Patient angegeben (Warkentin 2002). Die Laborkontrollen der Blutwerte sind nur in einem vergleichbar geringeren Umfang nötig (Weitz 1997). Studien zeigen weniger Blutungskomplikationen sowie eine geringere Gesamtmortalität (van der Belt et al. 2004). Im Vergleich zu den unfraktionierten Heparinen treten weniger thromboembolische Ereignisse auf (Dolovich et al. 2000). 
Die Antikoagulation wird durch die vermehrte Aktivierung von Antithrombin III erreicht. Es bewirkt eine Inaktivierung von Thrombin und aktivierten Faktor Xa (Greinacher 1997). Im Vergleich mit den unfraktionierten Heparinen bewirken die niedermolekularen Formen eine geringere Hemmung des Faktors Ila bei mindestens gleichstarker Hemmung des Faktors Xa. Die Applikation kann einmal oder zweimal täglich erfolgen, wobei in einem aktuellem Cochrane-Review die berechneten weiten 95\%Konfidenzintervalle ein möglicherweise größeres Risiko für eine Rezidiv-tVT bei Einmalgabe verdeutlichen (van Dongen et al. 2005).

Die Eliminierung erfolgt fast ausschließlich über die Nieren. Somit stellt deren Insuffizienz eine Kontraindikation für die Verwendung niedermolekularer Heparine dar (Schröder und Weiss 2002). Ebenso verbietet sich diese Art der Medikation bei Nachweis einer Heparin-induzierten Thrombozytopenie vom Typ II. Hierbei handelt es sich um einen immunologischen Prozess, bei dem es zwischen dem 5 . und 21. Tag zur Antikörperbildung kommt (Warkentin et al. 1995). Trotz des erheblichen Thrombozytenabfalls auf unter $50 \%$ des Ausgangswertes führt die Komplikation zu einer erhöhten Gerinnungsneigung (Warkentin und Greinacher 2004). Zu Beginn der Therapie sollte ein Ausgangswert der Blutplättchen bestimmt werden. Eine Überprüfung erfolgt im weiteren Verlauf der Heparingabe am 5. und 10. Tag. Danach sinkt das Risiko für das Auftreten einer HIT deutlich ab (Warkentin 2002).

\subsubsection{Frühmobilisation}

Das Konzept der strengen Bettruhe beruht auf der pathophysiologischen Annahme, dass bei Bewegung Thromben aus den Becken- und Beinvenen in die Körperstrombahn mobilisiert werden und Lungenembolien verursachen. Erst nachdem die Thromben nach ursprünglicher Annahme „organisiert“ sind, ging man von einem abnehmenden Risiko aus. Entgegen dieser historischen Vorgehensweise konnte in zahlreichen Studien gezeigt werden, dass die Mobilisierung des Patienten und die ambulante Therapieführung mindestens genauso sicher sind, wie die stationäre Behandlung (Koopman et al. 1996, Levine et al. 1996, Aschwanden et al. 2001, Schraibman et al. 2004).

Weitere Aspekte sind die deutliche Kostenreduktion und die gute Umsetzbarkeit in die Praxis (O`Brien et al. 1999). Aktuelle prospektive Studien konnten eine geringere Komplikationsrate bei ambulanter Therapieführung belegen (Offermann et al. 2001, 
Schwarz et al. 2001). Andere Autoren bestätigten die Möglichkeit der sicheren ambulanten Behandlung unabhängig von Ausmaß und Lokalisation der tiefen Beinvenenthrombose (Partsch und Blättler 2000). 


\section{$1.2 \quad$ Zielsetzung}

Die im Kapitel 1.1 beschriebenen Veränderungen in der Diagnostik und Therapie der tiefen Beinvenenthrombose führten zu folgenden Hypothesen:

1. Die Möglichkeit der frühen Mobilisation des Patienten und die Verwendung niedermolekularer Heparine führen zu einer Verlagerung der Diagnostik und Behandlung in den ambulanten Bereich.

2. Die unspezifische Klinik der tiefen Beinvenenthrombose erlaubt nach wie vor keine sichere Ausschlussdiagnose nur basierend auf der Symptomatik. Viele Patienten werden vom Hausarzt zum Spezialisten überwiesen, bei denen keine tVT nachgewiesen werden kann.

3. Die Vorteile der Farbduplexsonographie bewirken eine zunehmende Etablierung dieses Verfahrens. Die Phlebographie wird nur noch im reduzierten Umfang eingesetzt.

4. Die D-Dimer-Spiegelbestimmung wird zunehmend in den diagnostischen Prozess eingebunden.

Zur Beantwortung der Hypothesen werden Daten aus den an der Versorgung von Patienten mit phlebologischen Krankheitsbildern beteiligten Institutionen retrospektiv erfasst. Hierbei werden sowohl ambulante als auch stationär versorgende Einrichtungen berücksichtigt. Es sollen Unterschiede in der Vorgehensweise und ggf. Verschiebungen zwischen den beiden Bereichen beurteilt werden. Da die wesentlichen Änderungen der Diagnostik und Therapie den Jahren 1990 bis 2001 zuzuschreiben sind (s. Kapitel 1.1), wurde ein entsprechender Beobachtungszeitraum gewählt.

Die Studie soll klären, inwieweit die neuen diagnostischen und therapeutischen Möglichkeiten in den klinischen Alltag umgesetzt werden. Ein besonderer Fokus liegt auf der Verwendung der Duplexsonographie, der Etablierung der D-Dimer-Bestimmung sowie der Durchführung einer ambulanten Therapieform.

Die gewünschte Charakterisierung der vom Hausarzt zum Spezialisten mit tVTVerdacht überwiesenen Patienten konnte nur bedingt durchgeführt werden. Ursächlich war die unvollständige Dokumentation der initialen Symptomatik und der körperlichen Untersuchungsbefunde in den einbezogenen Institutionen (Hypothese 2). Daher führten wir eine ergänzende prospektive Untersuchung durch. Berücksichtigt 
wurden vom Hausarzt zum Spezialisten überwiesene Patienten. Auf der Basis eines neu konzipierten Erfassungsbogens konnte die Symptomatik und der körperliche Untersuchungsbefund standardisiert erfasst werden. Eine zuverlässige Datengrundlage ist somit gewährleistet. 


\section{Patienten und Methoden}

\subsection{Methodische Anlage der Untersuchung}

Zur Darstellung der Veränderungen in der Diagnostik und Therapie der tiefen Beinvenenthrombose (tVT) (Hypothesen 1,3 und $4 \mathrm{~s}$. Kapitel 1.2) wurden retrospektiv Daten erhoben. Um eine möglichst stabile longitudinale Beurteilung der Entwicklung zu erhalten, haben wir uns für den Landkreis Northeim entschieden. In dieser in sich abgeschlossenen Region gibt es lediglich ein Krankenhaus sowie eine große phlebologische Schwerpunktpraxis. Beide konnten in die Studie einbezogen werden. Die einzige radiologische Praxis befindet sich am Krankenhaus und wurde darüber eingeschlossen. Die an der Versorgung von Patienten mit Verdacht auf eine IVT beteiligten Institutionen konnten somit erfasst werden. Die abgegrenzte geographische Lage sowie die umfassende Präsenz medizinischer Einrichtungen erlauben die Annahme, dass die meisten Patienten in diesem Bereich sowohl diagnostiziert als auch therapiert werden. Ebenso können wir von einer stabilen Population im Beobachtungszeitraum ausgehen. In der Umgebung des Landkreises ist es im Untersuchungszeitraum zu keinen Veränderungen in der medizinischen Versorgungsstruktur gekommen.

Der Landkreis Northeim umfasst unter Berücksichtigung der benachbarten kleineren Ortschaften 34.000 Einwohner und liegt ca. 22 Kilometer von Göttingen, dem nächstgelegenen Oberzentrum entfernt. Das Krankenhaus ist eine Einrichtung der Regelversorgung mit 301 Planbetten.

Als Untersuchungsinstrument für die retrospektive Erhebung wurde ein standardisierter Fragebogen (siehe Anhang) verwendet. Er erfasst die im Kapitel 2.2.2.1 beschriebenen Daten und wurde auf der Basis aktueller Literatur aus den Bereichen Lehre und Forschung entwickelt. Zusätzlich wurden die Erfahrungswerte der behandelnden Ärzte berücksichtigt.

Eine ergänzende prospektive Untersuchung sollte in einem primärärztlichen Patientenkollektiv überprüfen, inwieweit die körperliche Untersuchung den Ausschluss oder die Diagnose einer tVT zulässt (s. Hypothese 2, Kapitel 1.2). Die Inzidenz throm- 
boembolischer Ereignisse ist in der hausärztlichen Versorgung nicht sicher bekannt. Sie dürfte sich in einer Größenordnung von ca. 1-3/1000 bewegen. Somit wäre der logistische Aufwand der Erfassung von Patienten mit Verdacht auf eine IVT bei Hausärzten als sehr groß anzunehmen (Silverstein et al. 1998, Kearon 2001). Der Einschluss einer Vielzahl von Praxen und ein langer Beobachtungszeitraum wären erforderlich, um eine ausreichende Fallzahl zu erzielen. Daher wurden hausärztlich mit tVT-Verdacht überwiesene Patienten erfasst. Als Institution wurde die bereits im Rahmen der retrospektiven Untersuchung einbezogene phlebologische Schwerpunktpraxis gewählt. Bei der Auswertung darf nicht unberücksichtigt bleiben, dass es sich somit um eine bereits durch Hausärzte vorselektierte Gruppe von Patienten handelt. Für die prospektive Datenerhebung wurde ein spezieller Dokumentationsbogen eingesetzt. Er geht detailliert auf Patientensymptome und die ärztliche Vorgehensweise ein (siehe Anhang). Die Erfassung erfolgte in beiden Erhebungen anonym. Zur Identifikation wurde sowohl in der Praxis als auch im Krankenhaus auf die interne Identifikationsnummer zurückgegriffen.

Die Untersuchungszeiträume waren die Doppeljahrgänge 1990/1, 1995/6 und 2000/1. Diesem Zeitraum schreiben wir wesentliche Veränderungen in der Diagnostik und Therapie zu. Deren Umsetzung wollten wir mit dieser Studie abbilden (s. Kapitel 1.1). Die ergänzende prospektive Untersuchung stützt sich auf die Daten aus dem Zeitraum August 2001 bis April 2003.

\section{$2.2 \quad$ Retrospektiver Teil}

\subsubsection{Teilnehmende Patienten und medizinische Einrichtungen}

\subsubsection{Phlebologische Schwerpunktpraxis}

Im Schnitt wurden im Untersuchungszeitraum pro Jahr ca. 8000 Patienten behandelt. Bezogen auf den gesamten Untersuchungszeitraum lag der Anteil der relevanten Thrombosefälle bei ca. 0,9\%. Die genauen Zahlen sind der Tabelle 3 zu entnehmen. Deutlich ist ein Anstieg der an einer tiefen Beinvenenthrombose Erkrankten im Verhältnis zur Gesamtpatientenzahl über den Erfassungszeitraum zu erkennen (Erhebung der Daten siehe Kapitel 2.2.3.1). 
Tabelle 3: Summe der Patienten mit Verdacht auf eine tiefe Beinvenenthrombose im Verhältnis zu allen Patienten der Praxis

\begin{tabular}{llcc}
\hline & Patienten gesamt & V.a. Thrombose & V.a. Thrombose/Patienten (in \%) \\
\hline $\mathbf{1 9 9 1}$ & 6172 & 27 & 0,44 \\
$\mathbf{1 9 9 2}$ & 6941 & 33 & 0,48 \\
$\mathbf{1 9 9 3}$ & 7074 & 29 & 0,41 \\
$\mathbf{1 9 9 4}$ & 7434 & 45 & 0,61 \\
$\mathbf{1 9 9 5}$ & 8783 & 71 & 0,81 \\
$\mathbf{1 9 9 6}$ & 9291 & 51 & 0,55 \\
$\mathbf{1 9 9 7}$ & 9744 & 116 & 1,19 \\
$\mathbf{1 9 9 8}$ & 8225 & 101 & 1,23 \\
$\mathbf{1 9 9 9}$ & 8490 & 68 & 0,80 \\
$\mathbf{2 0 0 0}$ & 8692 & 110 & 1,27 \\
$\mathbf{2 0 0 1}$ & 8549 & 112 & 1,31 \\
\hline
\end{tabular}

Die Angaben beziehen sich auf alle Patienten mit Verdacht auf eine tiefe Beinvenenthrombose pro Jahr (Abb. 1). Die Untersuchung der Patientenakten begrenzte sich auf die Doppeljahrgänge 1990/1, 1995/6 und 2000/1.

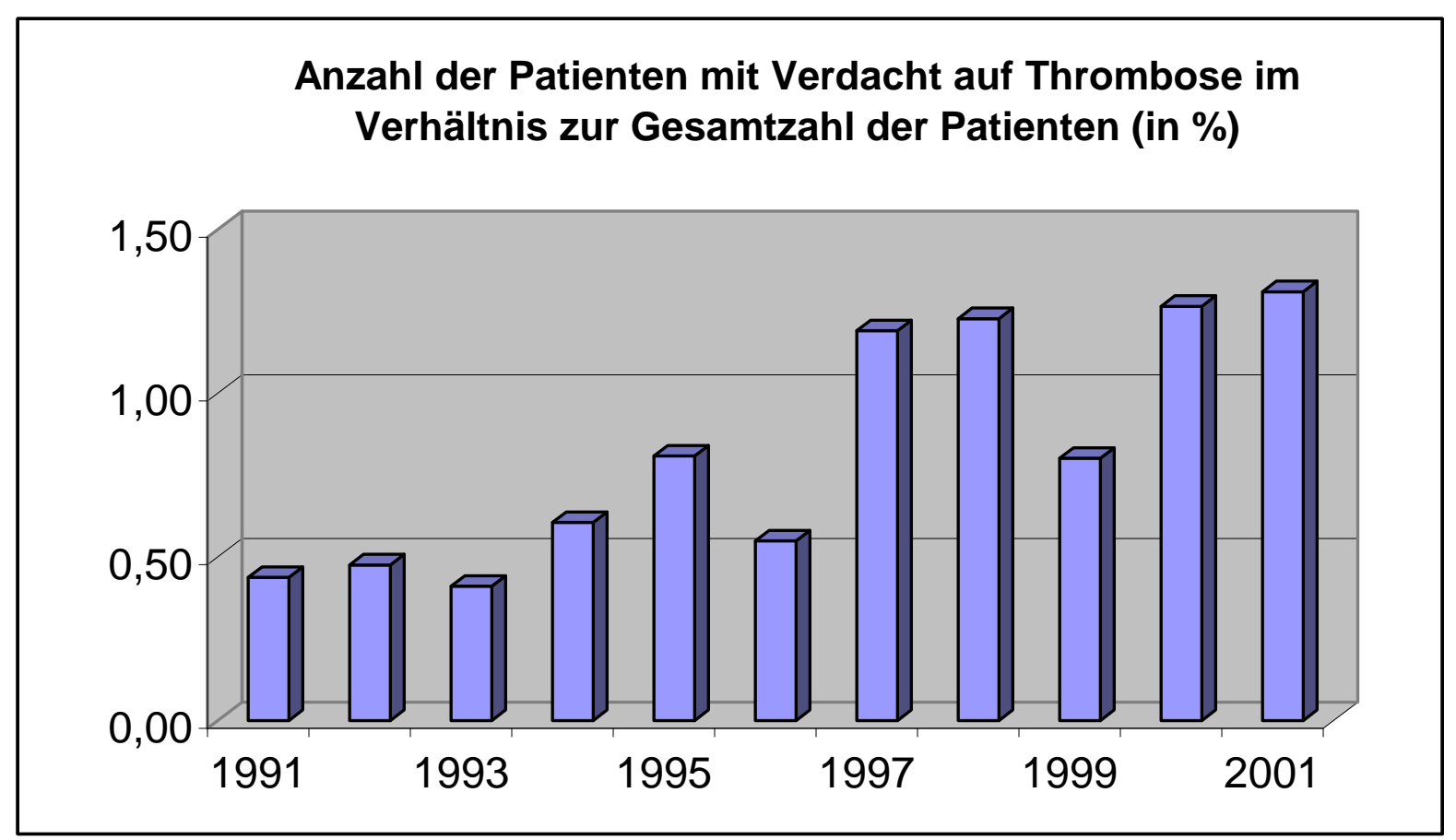

Abb. 1: Zunahme der tVT-Verdachtsfälle in der phlebologischen Schwerpunktpraxis im Untersuchungszeitraum 
Nach Rücksprache mit den jeweilig behandelnden Ärzten ließ sich ermitteln, dass alle Patienten mit tVT-Verdacht üblicherweise in der Praxis-EDV entweder im Freitext oder als ICD-Kodierung verwaltet wurden. Die Suchstrategie zur Identifikation der Patienten beinhaltete demnach eine EDV-Abfrage mit dem Suchbegriff „Thrombose“ und der dazu passenden ICD-Verschlüsselung. So konnten alle in Frage kommenden Patienten erfasst werden Abfragen nach Suchbegriffen innerhalb der Freitextangaben ermöglichten die Erfassung der Patienten, bei denen lediglich der „Verdacht auf" bestand. Dieses sehr sensitive aber wenig spezifische Vorgehen erfasste auch Patienten z.B. mit einer Perianalvenenthrombose. Hier erfolgte im Rahmen der anschließenden Handsuche in den Karteikarten eine Zuordnung beziehungsweise Ausschluss. Für die Studie wichtige Angaben wurden den Akten entnommen und auf den Erfassungsbögen dokumentiert. Die Anzahl der insgesamt an der Untersuchung beteiligten Patienten belief sich auf 371.

\subsubsection{Krankenhaus}

Das Albert-Schweitzer-Krankenhaus in Northeim wurde ebenfalls in die Studie aufgenommen. Thrombosen, die erstmalig während einer stationären Behandlung auftraten, fanden keine Berücksichtigung. Die Arbeit untersucht nur die von extern zugewiesenen Patienten.

Im Gegensatz zur Datenerhebung in der Praxis erwies sich die Erfassung im Krankenhaus als sehr problematisch. Ursächlich war die erst ab dem Jahr 2001 vorhandene Möglichkeit der Patientenverwaltung mittels EDV. Alle Patienten mit tVTVerdacht wurden jedoch gemäß einer Dienstanweisung duplexsonographisch untersucht. Über die erfolgte Dokumentation der Untersuchung ließen sich die relevanten Patienten erfassen. Auf Grund von Archivierungsproblemen (Umstellung der Karteisysteme auf EDV, Auslagerung von Papierakten nach extern) konnten die identifizierten Patienten der Jahre 1990/1, 1995 und 2000 trotz erheblicher Anstrengungen der Beteiligten nur sehr unvollständig als Papierkartei eingesehen werden. Daher musste sich die Untersuchung auf die vollständig zu erfassenden Jahrgänge 1996 und 2001 beschränken. Trotz dieser Problematik ließ sich eine hohe Fallzahl an Patienten mit tVT-Verdacht ermitteln. So konnten 710 Krankenhauspatienten in die Studie einfließen (1996: 257 Patienten, 2001: 453). 


\subsubsection{Untersuchungsinstrument}

Für die Erfassung der Praxisdaten wurde ein standardisierter Fragebogen verwendet, der sich inhaltlich in acht Themenblöcke gliedert (siehe Anhang). Die Dokumentation relevanter Daten erfolgte mittels freier Texte und in Form des Ankreuzens in einer vorher bestehenden Auswahl von Antwortmöglichkeiten. Der zwei Din-A4Seiten umfassende Bogen enthielt neben soziodemographischen Angaben folgende Unterpunkte:

- Die wichtigsten Vorerkrankungen und Risikofaktoren wurden erfasst, die das Auftreten einer tiefen Beinvenenthrombose begünstigen. Neben einer eventuell vorausgegangenen Immobilität oder Verletzung zählten hierzu Begleitumstände wie Nikotinabusus, bestehende Gravidität, orale Kontrazeption, eine arterielle Hypertonie oder die Erkrankung an Diabetes mellitus. Auch ein gegebenenfalls vorhandenes Tumorleiden wurde dokumentiert.

- Da die Einnahme gerinnungshemmender Medikamente starken Einfluss auf die Fließeigenschaften des Blutes hat, wurde eine in diesem Punkt bestehende Vormedikation in die Studie aufgenommen.

- Angaben bezüglich der patienteneigenen Thromboseanamnese konnten ebenso erfasst werden, wie die Daten der Familienanamnese. Berücksichtigung fanden hierbei der Zeitpunkt und die genaue Lokalisation der Erkrankung sowie das jeweilige Ergebnis der Untersuchung.

- Im gleichen Umfang wurden die Daten der Thrombophilieanamnese dokumentiert.

- Um Häufigkeiten feststellen zu können, sind Angaben betreffend der akuten Symptomatik und vorhandener Untersuchungszeichen erfasst worden. Hierzu gehörten die Leitsymptome der tiefen Beinvenenthrombose wie Schmerzen, Schwellungen und Verfärbungen der betroffenen Extremität.

- Wichtige Untersuchungsmerkmale waren die Umfangsdifferenz, die Überwärmung sowie der Wadendruckschmerz.

- Die Erfassung der Veränderungen in der apparativen Thrombosediagnostik war ein weiteres Ziel dieser Studie. Der Stellenwert der Duplexsonographie als auch der Phlebographie in den einzelnen Untersuchungszeiträumen sollte er- 
fasst werden. Der Einsatz der D-Dimer-Bestimmung wurde in die Studie integriert.

- Bei der Dokumentation der Diagnose wurde zwischen sicherer, eventuell vorhandener und eindeutig ausgeschlossener Thrombose unterschieden. Unterteilt wurde die Lokalisation bei den nachgewiesenen und den Verdachtsfällen in Becken, Oberschenkel und Unterschenkelthrombose. Angaben bezüglich einer Lungenembolie als wichtige Begleit- bzw. Folgeerkrankung sind berücksichtigt worden.

- Therapeutisch wurde grundsätzlich zwischen einem ambulanten und stationären Vorgehen unterschieden. Wichtige Behandlungsmöglichkeiten der tiefen Beinvenenthrombose konnten erfasst werden. Hierzu zählten die Durchführung einer Lyse, die Verordnung eines niedermolekularen oder unfraktionierten Heparins sowie die Anwendung eines Vitamin K Antagonisten. Angaben im Hinblick auf eine Kompressionstherapie und deren Stärke und Dauer wurden dokumentiert. Falls die Aktenlage einen Hinweis auf die Mobilisierung des Patienten ergab, ist dies auf den Erfassungsbögen notiert worden.

- Berücksichtigung fanden die Anzahl der angefertigten Entlassungsbriefe sowie deren Inhalte. Hierzu gehörten neben der Angabe über den Zeitpunkt der Erstellung des Briefes nach Entlassung Daten, aus denen die medikamentöse Weiterbehandlung des Patienten hervorging. Erfasst wurde die Art und Dauer der Behandlung mit Phenprocoumon und Heparin. Bei letzterem war vor allem die Entscheidung zwischen einem niedermolekularen und einem unfraktionierten Heparin von Interesse. Die Verordnung einer Kompression sowie deren Dauer und Stärke, die Durchführung und die Ergebnisse der Tumor- und Thrombophiliediagnostik wurden ausgewertet.

- Die Auswertung des Krankheitsverlaufes beinhaltete neben der eigentlichen Anzahl an dokumentierten Kontrollen die Erfassung von Blutungen, Rezidiven und Lungenemboliezeichen. Hinweise auf ein postthrombotisches Syndrom wurden vermerkt.

\subsection{Prospektiver Teil}

Die prospektive Datenerfassung in der phlebologischen Schwerpunktpraxis umfasste den Zeitraum von August 2001 bis April 2003. Alle hausärztlich überwiesenen Pati- 
enten mit Thromboseverdacht wurden in die Studie aufgenommen. Die Praxis-EDV diente zum Abgleich, um eine Vollständigkeit der Erfassung zu gewährleisten. Mittels eines vorher erstellten Erhebungsbogens wurden sämtliche Patienten standardisiert befragt und ihre Anamnese und Beschwerdesymptomatik dokumentiert (siehe Anhang). Das jeweilige Ergebnis der körperlichen und apparativen Untersuchung konnte hierüber erfasst werden.

Die Untersuchung der Patienten beinhaltete (gemäß dem derzeitigen internen Praxisstandard) eine Farbduplexsonographie des venösen Gefäßsystems der Beine (entsprechend den zum Untersuchungszeitpunkt aktuellen Empfehlungen der Leitlinie der Deutschen Gesellschaft für Phlebologie) (Blättler et al. 1998). Auf Grund der persönlichen Erfahrung der behandelnden Ärzte (mündliche Mitteilung), dass gerade im distalen Bereich bei der Farbduplexsonographie eine relevante Anzahl an falschpositiven Diagnosen auftritt, wurde (in Abweichung von der genannten Leitlinie) bei sonographischem Verdacht auf eine tVT eine anschließende Phlebographie zur Diagnosesicherung und Dokumentation der Ausdehnung veranlasst. Ließ sich sonographisch nicht sicher eine tiefe Beinvenenthrombose ausschließen, wurde bei niedriger klinischer tVT-Wahrscheinlichkeit zur weiteren Diagnostik eine D-DimerSpiegelbestimmung durchgeführt. Bei mittlerer und hoher klinischer Wahrscheinlichkeit folgte eine phlebographische Untersuchung. Diese Vorgehensweise deckt sich mit derjenigen aktueller Studien (Blättler et al. 1998, Kearon et al. 2001) Das genaue diagnostische Procedere wird in der Abbildung 2 (s. Kapitel 3.13) dargestellt.

\subsection{Datenanalyse}

Die Erfassung der Daten erfolgte mit der Datenbank dBase in der Version 5.1.

Für die statistische Auswertung der erhobenen Daten wurde das Programm SAS für Windows in der Version 8.2 verwendet (SAS Institute 1999). 


\section{Ergebnisse}

Die Ergebnisse in den Abschnitten 3.1-3.12 beziehen sich auf die retrospektiven Daten.

\subsection{Erfasste Patienten}

Bei Anwendung der in Kapitel 2 besprochenen Kriterien wurden insgesamt 371 Patientenakten in der Praxis eingesehen. Sie verteilten sich wie folgt auf die untersuchten Doppeljahrgänge: 27 Akten wurden für die Jahre 1990/1 retrospektiv ausgewertet, 1995/96 waren es 122 Akten und 2000/01 222 Akten.

Im Krankenhaus wurden 257 Dokumente für den Zeitraum 1996 bearbeitet. 2001 belief sich die Summe der ausgewerteten Akten auf 453.

\subsection{Beschreibung der Stichprobe}

\subsubsection{Praxis}

Ein Überblick über das gesamte Patientenkollektiv der Praxis und den darin anteilig enthaltenen Thrombosefällen ist der Tabelle 3 in Kapitel 2.2.1.1 zu entnehmen. Es ist das Verhältnis der Patienten mit Thromboseverdacht zu allen Patienten der Praxis angegeben. Im Vierfeldertest zeigt sich ein signifikanter Anstieg der Verdachtsfälle im Vergleich der Jahre 2001 und $1991\left(\chi^{2}=36,7, p<0,01\right)$.

Der Tabelle 4 ist die Verteilung weiblicher und männlicher Patienten im prozentualen Vergleich in den drei untersuchten Doppeljahrgängen zu entnehmen. Dabei konnte statistisch im Mehrfeldertest sowohl für alle 3 Doppeljahrgänge zusammen ( $\chi 2=3,53$, $p=0,17$ ) als auch für die Doppeljahrgänge untereinander (im Vierfeldertest) kein signifikanter Unterschied nachgewiesen werden (1990/1 vs. 1995/6: $p=0,052 ; 1995 / 6$ vs. 2000/1: $p=0,089$ ).

Im Vergleich der Doppeljahrgänge 1990/1 und 1995/6 zeigte sich eine signifikant ältere Population in den Jahren 1995/6 ( $t=2,28, p=0,024)$. Zwischen den Jahrgängen 1995/6 und 2000/1 fand sich kein Unterschied $(p=0,78)$ (Tabelle 4). 
Tabelle 4: Geschlechterverteilung und Alter des erfassten Patientenkollektivs in der Praxis (Alter als arithmetischer Mittelwert sowie Standardabweichung; die Prozentangaben beziehen sich auf die Gesamtzahl aller Patienten pro Doppeljahrgang)

\begin{tabular}{lccc}
\hline & $\mathbf{1 9 9 0 / 9 1}$ & $\mathbf{1 9 9 5 / 9 6}$ & $\mathbf{2 0 0 0 / 0 1}$ \\
\hline gesamt & 27 & 122 & 222 \\
weiblich & $23(85,2 \%)$ & $85(69,7 \%)$ & $150(67,6 \%)$ \\
männlich & $4(14,8 \%)$ & $37(30,3 \%)$ & $72(32,4 \%)$ \\
Altersdurchschnitt & $46,2( \pm 15,9)$ & $54,4( \pm 17,0)$ & $53,9( \pm 16,1)$ \\
\hline
\end{tabular}

\subsubsection{Krankenhaus}

Die entsprechenden Daten der beiden untersuchten Jahre aus dem Krankenhaus gehen aus der Tabelle 5 hervor. Statistisch fand sich hier im Vierfeldertest ein signifikanter Unterschied in der Geschlechterverteilung $\left(\chi^{2}=4,79, p=0,029\right)$. Bezüglich des Alters fand sich im $t$-Test kein signifikanter Unterschied ( $t=1,27, p=0,203)$.

Tabelle 5: Geschlechterverteilung und Alter der im Krankenhaus erfassten Patientendaten (Alter als arithmetischer Mittelwert sowie Standardabweichung; die Prozentangaben beziehen sich auf alle relevanten Patienten pro Jahr)

\begin{tabular}{lll}
\hline & $\mathbf{1 9 9 6}$ & $\mathbf{2 0 0 1}$ \\
\hline gesamt & 257 & 453 \\
weiblich & $155(60,3 \%)$ & $310(68,4 \%)$ \\
männlich & $102(39,7 \%)$ & $143(31,6 \%)$ \\
Altersdurchschnitt & & \\
\hline
\end{tabular}

\subsection{Vorerkrankungen und Risikofaktoren}

Es wurden die wichtigsten Faktoren erfasst, die das Auftreten einer tiefen Beinvenenthrombose begünstigen. Eine Auflistung ist der Tabelle 6 zu entnehmen. Da bei keinem Patienten eine relevante längere Immobilität oder Reise anamnestisch festgestellt werden konnte, ist dieser Punkt nicht in der Tabelle aufgeführt. 
Tabelle 6: Risikofaktoren die das Auftreten einer tiefen Beinvenenthrombose begünstigen (Absolutzahlen und prozentuale Häufigkeit pro Doppeljahrgang; Daten der Praxis)

\begin{tabular}{lccccc}
\hline & \multicolumn{1}{l}{$\mathbf{1 9 9 0 / 9 1}$} & \multicolumn{2}{l}{$\mathbf{1 9 9 5 / 9 6}$} & \multicolumn{2}{l}{$\mathbf{2 0 0 0 / 0 1}$} \\
\hline Diabetes mellitus & 2 & $(7,4 \%)$ & 2 & $(1,6 \%)$ & $5(2,3 \%)$ \\
Raucher & 1 & $(3,7 \%)$ & 0 & $(0,0 \%)$ & $8(3,6 \%)$ \\
art. Hypertonie & 3 & $(11,1 \%)$ & 4 & $(3,3 \%)$ & $11(5,0 \%)$ \\
Tumor & 0 & $(0,0 \%)$ & 1 & $(0,8 \%)$ & $9(4,1 \%)$ \\
Verletzung & 3 & $(11,1 \%)$ & 9 & $(7,4 \%)$ & $12(5,4 \%)$ \\
Varikosis & 0 & $(0,0 \%)$ & 5 & $(4,1 \%)$ & $7(3,2 \%)$ \\
orale Kontrazeptiva & 1 & $(3,7 \%)$ & 2 & $(1,6 \%)$ & $1(0,5 \%)$ \\
Schwangerschaft & 0 & $(0,0 \%)$ & 1 & $(0,8 \%)$ & $0(0,0 \%)$ \\
\hline
\end{tabular}

\subsection{Thromboseanamnese}

\subsubsection{Praxis}

Im Doppeljahrgang 1990/91 konnte bei 8 (29,6\%) Patienten eine positive Thromboseanamnese erhoben werden. In den Jahren 1995/6 belief sich die Anzahl auf 20 (16,4\%). 2000/01 lag eine positive Thromboseanamnese bei 45 (20,3\%) Patienten vor, von denen 38 einmal und 7 Patienten zweimal an einer tiefen Beinvenenthrombose erkrankt waren.

\subsubsection{Krankenhaus}

Bei 26 (10,1\%) Patienten aus dem Jahr 1996 war den Akten eine positive Thromboseanamnese zu entnehmen. 2001 waren 89 (19,6\%) Patienten schon einmal an einer tiefen Beinvenenthrombose erkrankt.

\subsection{Thrombophilie}

Gerinnungsstörungen können zur Entwicklung tiefer Beinvenenthrombosen beitragen. Die entsprechenden Daten der Praxis sind der Tabelle $7 \mathrm{zu}$ entnehmen. Berücksichtigt wurde die Summe der Patientenzahl pro Doppeljahrgang, die eine Thrombophilie aufwies. 
Tabelle 7: Summe vorhandener Thrombophilien in der Anamnese (Absolutzahlen und prozentualer Anteil am Gesamtkollektiv des entsprechenden Doppeljahres in der Praxis)

\begin{tabular}{|c|c|c|c|}
\hline Daten - Thrombophilie & $1990 / 91$ & $1995 / 96$ & $2000 / 01$ \\
\hline vorhanden & $1 \quad(3,7 \%)$ & $11 \quad(9,0 \%)$ & $11 \quad(5,0 \%)$ \\
\hline davon positiv & 1 & 10 & 9 \\
\hline davon negativ & 0 & 1 & 2 \\
\hline nicht vorhanden & $26(96,3 \%)$ & $111(91,0 \%)$ & $211(95,0 \%)$ \\
\hline
\end{tabular}

\subsection{Vormedikation}

Die Patientenakten der Praxis wurden dahingehend untersucht, ob bereits eine Vormedikation mit Antikoagulantien bestand.

1990/1 nahm ein (3,7\%) Patient Acetylsalicylsäure (ASS) ein, zwei (7,4\%) weitere verwendeten Phenprocoumon.

1995/6 konnte den Akten bei 2 (1,6\%) Patienten eine ASS-Einnahme und bei 5 $(4,1 \%)$ Patienten eine Phenprocoumontherapie entnommen werden.

2000/1 zeigte sich anhand der Dokumentation eine ASS - Medikation bei 12 (5,4\%) Patienten und eine Vitamin K-Antagonisierung bei 11 (5,0\%) Patienten.

\subsection{Symptomatik zum Untersuchungszeitpunkt}

Tabelle 8 veranschaulicht die Häufigkeit der dokumentierten Krankheitszeichen, die auf eine tiefe Beinvenenthrombose schließen lassen. Die Auswertung der Praxisunterlagen ergab, dass die Schwellung der betroffenen Extremität das am häufigsten beschriebene Symptom war. Ebenso standen häufig Schmerzen im Vordergrund des Beschwerdebildes. Unter Sonstiges sind Krankheitszeichen wie Brennen, Jucken, Verhärtungen und Sensibilitätsstörungen zusammengefasst. Bedingt durch das gleichzeitige Auftreten mehrerer Symptome liegt die Summe der Prozentangaben zum Teil bei über $100 \%$. 
Tabelle 8: Art und Häufigkeit der Symptomatik (Anzahl der Patienten absolut und in Prozent bezogen auf das entsprechende Doppeljahr; Daten der Praxis)

\begin{tabular}{lcccccc}
\hline & \multicolumn{1}{c}{$1990 / \mathbf{1}$} & \multicolumn{1}{c}{$\mathbf{1 9 9 5 / 6}$} & \multicolumn{2}{c}{$\mathbf{2 0 0 0 / 1}$} \\
\hline Schwellung & 15 & $(55,6 \%)$ & 84 & $(68,9 \%)$ & 147 & $(66,2 \%)$ \\
Schmerzen & 12 & $(44,4 \%)$ & 48 & $(39,3 \%)$ & 149 & $(67,1 \%)$ \\
Verfärbung & 0 & $(0,0 \%)$ & 3 & $(2,5 \%)$ & 2 & $(0,9 \%)$ \\
Symptome LE & 0 & $(0,0 \%)$ & 0 & $(0,0 \%)$ & 0 & $(0,0 \%)$ \\
Sonstiges & 1 & $(3,7 \%)$ & 14 & $(11,5 \%)$ & 10 & $(4,5 \%)$ \\
\hline
\end{tabular}

(LE: Lungenembolie)

\subsection{Untersuchungsbefunde}

Bei der körperlichen Untersuchung zeigte sich, dass über den gesamten Zeitraum die Umfangsdifferenz am häufigsten diagnostiziert wurde. Bezogen auf die Gesamtzahl aller in einem Doppeljahrgang vorgekommenen tiefen Beinvenenthrombosen lag der prozentuale Anteil einer gleichzeitig vorhandenen Umfangsdifferenz in den Jahren $1990 / 1$ bei $90 \%, 1995 / 6$ bei $78,4 \%$ und $2000 / 1$ bei $100 \%$. Eine Überwärmung ließ sich $1990 / 1$ nur bei $10 \%, 1995 / 6$ bei $15,7 \%$ und $2000 / 1$ bei $21,3 \%$ der IVT - Patienten nachweisen. Unter Beachtung dieser Kriterien lag das prozentuale Vorkommen des Wadendruckschmerzes $1990 / 1$ bei $80 \%, 1995 / 6$ bei $11,8 \%$ und $2000 / 1$ bei $55,7 \%$.

Eine Übersicht der am zahlreichsten durchgeführten Untersuchungen gibt Tabelle 9.

Tabelle 9: Häufigkeit durchgeführter Untersuchungen in der Praxis (Die Prozentangaben beziehen sich auf alle erfassten Patienten pro Doppeljahrgang. Da Mehrfachnennungen möglich waren, kann die Summe mehr als $100 \%$ betragen.)

\begin{tabular}{|c|c|c|c|}
\hline & 1990/1 & $1995 / 6$ & $2000 / 1$ \\
\hline Umfangsdifferenz & $9(33,3 \%)$ & $40 \quad(32,8 \%)$ & $73(32,9 \%)$ \\
\hline Überwärmung & $1 \quad(3,7 \%)$ & $8 \quad(6,6 \%)$ & $13 \quad(5,9 \%)$ \\
\hline Wadendruckschmerz & $8 \quad(29,6 \%)$ & $6 \quad(4,9 \%)$ & $34 \quad(15,3 \%)$ \\
\hline
\end{tabular}




\subsection{Diagnostik}

\subsubsection{Praxis}

Die Tabelle 10 zeigt eine Aufstellung der apparativen Diagnostik. Auffällig ist die prozentuale Halbierung der durchgeführten Phlebographien (1990/1 vs. 2000/1: $\left.\chi^{2}=27,61, p<0,0001\right)$. Der Anteil der Duplexsonographien erhöhte sich im Untersuchungszeitraum kontinuierlich (1990/1 vs. $1995 / 6: \chi^{2}=20,73, p<0,0001 ; 1995 / 6$ vs. 2000/1: $\left.\chi^{2}=7,167, p=0,0074\right)$. Kompressionssonographien wurden über den gesamten Untersuchungszeitraum nicht angewendet. Die Bestimmung der D-Dimere hat erst in den Jahren 2000/1 an Bedeutung gewonnen.

Tabelle 10: Anwendung diagnostischer Möglichkeiten und deren prozentualer Anteil an der Gesamtzahl der Patienten pro Jahr (Daten der Praxis; Mehrfachnennungen waren möglich, so dass die Summe bei über 100\% liegen kann)

\begin{tabular}{|c|c|c|c|c|c|c|}
\hline \multirow[b]{2}{*}{ D-Dimere bestimmt } & \multicolumn{2}{|c|}{$1990 / 91$} & \multicolumn{2}{|c|}{$1995 / 96$} & \multicolumn{2}{|c|}{ 2000/01 } \\
\hline & 0 & $(0,0 \%)$ & 0 & $(0,0 \%)$ & 7 & $(3,2 \%)$ \\
\hline davon positiv & 0 & $(0,0 \%)$ & 0 & $(0,0 \%)$ & 1 & $(0,5 \%)$ \\
\hline Duplexsonographie durchgeführt & 19 & $(70,4 \%)$ & 119 & $(97,5 \%)$ & 216 & $(97,3 \%)$ \\
\hline davon positiv & 3 & $(11,1 \%)$ & 29 & $(23,8 \%)$ & 30 & $(13,5 \%)$ \\
\hline Phlebographie durchgeführt & 12 & $(44,4 \%)$ & 34 & $(27,9 \%)$ & 49 & $(22,1 \%)$ \\
\hline davon positiv & 5 & $(18,5 \%)$ & 17 & $(13,9 \%)$ & 19 & $(8,6 \%)$ \\
\hline
\end{tabular}

\subsubsection{Krankenhaus}

Die Aktenlage des Krankenhauses ermöglichte die Erfassung der stattgehabten Duplexsonographien und Phlebographien. Im Jahr 1996 wurde bei zwei (0,8\%) Patienten eine Phlebographie durchgeführt. 2001 waren es 21 (4,6\%) Patienten. Eine Ultraschalluntersuchung wurde bei allen relevanten Patienten angewendet, die sich in den Jahren 1996 und 2001 im Krankenhaus vorstellten. 


\subsection{Diagnosestellung}

\subsubsection{Praxis}

In Tabelle 11 wird der prozentuale Anteil der tiefen Beinvenenthrombosen am Gesamtkollektiv pro Jahr beziehungsweise Doppeljahrgang gezeigt. Die separate Darstellung der Jahre 1995/96 und 2000/01 erlaubt einen besseren Vergleich mit den ermittelten Daten aus dem Krankenhaus. Bei insgesamt sechs Patienten ließ sich nicht eindeutig eine tiefe Beinvenenthrombose ausschließen (Verdachtsfälle). Sie wurden zur Sicherheit wie eine IVT behandelt. Bei der Beurteilung der ermittelten Häufigkeiten lässt sich bei einer Abnahme der positiven tVT-Diagnosen, eine leichte Zunahme der Thromboseausschlüsse im Beobachtungszeitraum 1995/6 verglichen mit $2000 / 1$ erkennen ( $41,8 \%$ vs. $\left.27,6 \% ; \chi^{2}=7,21, p=0,007\right)$. Für die Zeiträume $1990 / 1$ verglichen mit $1995 / 6$ fand sich demgegenüber kein Unterschied (38,5\% vs. $41,8 \%$; $\left.\chi^{2}=0,099, p=0,753\right)$. Die Abbildung 2 verdeutlicht die Häufigkeit der gestellten Diagnosen im Verhältnis zur Gesamtzahl.

Tabelle 11: Absolutzahlen und Prozentangaben der gesicherten Diagnose einer tiefen Beinvenenthrombose im Vergleich zu den Ausschlüssen und den Verdachtsfällen (Daten der Praxis)

\begin{tabular}{lcccccccccc}
\hline & $1990 / 91$ & \multicolumn{1}{c}{1995} & \multicolumn{1}{c}{1996} & \multicolumn{2}{c}{2000} & \multicolumn{2}{c}{2001} \\
\hline tVT positiv & 10 & $(37,0 \%)$ & 24 & $(33,8 \%)$ & 27 & $(52,9 \%)$ & 28 & $(25,5 \%)$ & 33 & $(29,5 \%)$ \\
tVT Ausschluss & 17 & $(63,0 \%)$ & 46 & $(64,8 \%)$ & 23 & $(45,1 \%)$ & 79 & $(71,8 \%)$ & 78 & $(69,6 \%)$ \\
Verdacht auf tVT & 0 & $(0,0 \%)$ & 1 & $(1,4 \%)$ & 1 & $(2,0 \%)$ & 3 & $(2,7 \%)$ & 1 & $(0,9 \%)$ \\
gesamt & $27(100,0 \%)$ & 71 & $(100,0 \%)$ & 51 & $(100,0 \%)$ & 110 & $(100,0 \%)$ & $112(100,0 \%)$ \\
(tVT: tiefe Beinvenenthrombose) & & & & & & &
\end{tabular}

Prozentangaben der gesicherten Diagnose im Vergleich zu den Ausschlüssen und den Verdachtsfällen

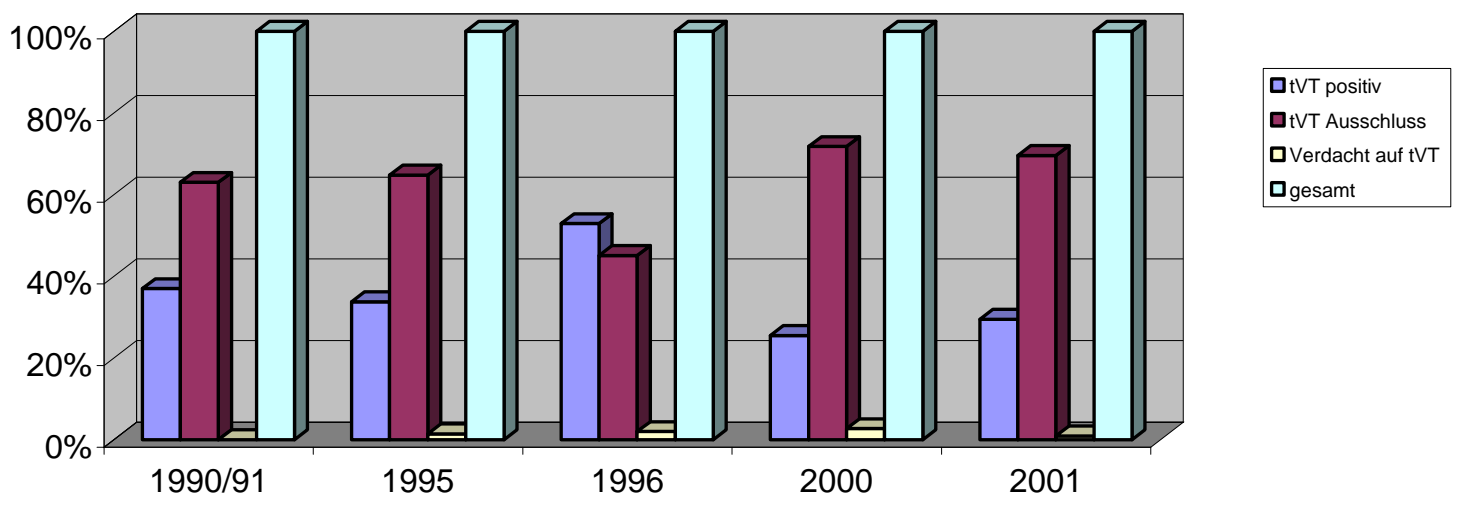

Abb.2: Diagnosen in der Praxis im Verhältnis zur Gesamtzahl (in \%) 
In Tabelle 12 sind die Lokalisationen der tiefen Beinvenenthrombosen angegeben. Die Abweichung zur Gesamtzahl der diagnostizierten Thrombosen in Tabelle 11 ergibt sich aus der nicht vollständigen Dokumentation bei drei Patienten. Hier fehlte die Angabe über die betroffene Beinregion. Die Prozentangaben beziehen sich auf die Gesamtzahl der positiven Diagnosen im entsprechenden Jahr.

Tabelle 12: Lokalisation der tiefen Beinvenenthrombose in der Praxis

\begin{tabular}{|c|c|c|c|c|c|c|c|c|c|}
\hline \multirow[b]{2}{*}{ Becken } & $1990 / 91$ & \multicolumn{2}{|c|}{1995} & \multicolumn{2}{|c|}{1996} & \multicolumn{2}{|c|}{2000} & \multicolumn{2}{|c|}{2001} \\
\hline & $3(30,0 \%)$ & 0 & $(0,0 \%)$ & 4 & $(14,8 \%)$ & 1 & $(3,6 \%)$ & 0 & $(0,0 \%)$ \\
\hline Oberschenkel & $1 \quad(10,0 \%)$ & 5 & $(20,8 \%)$ & 6 & $(22,2 \%)$ & 5 & $(17,9 \%)$ & 2 & $(6,1 \%)$ \\
\hline Unterschenkel & $6 \quad(60,0 \%)$ & 18 & $(75,0 \%)$ & 17 & $(63,0 \%)$ & 21 & $(75,0 \%)$ & 30 & $(90,9 \%)$ \\
\hline
\end{tabular}

\subsubsection{Krankenhaus}

Die Patientenzahl hat im Untersuchungszeitraum zugenommen. Der Tabelle 13 ist eine Übersicht über die Häufigkeit tiefer Beinvenenthrombosen zu entnehmen. Der relative Anteil der nachgewiesenen Thrombosen blieb nahezu konstant $\left(\chi^{2}=0,925\right.$, $\mathrm{p}=0,336$ ).

Tabelle 13: Verteilung des Gesamtkollektivs auf gesicherte und ausgeschlossene tiefe Beinvenenthrombose - absolut und prozentual angegeben (im Krankenhaus)

\begin{tabular}{lrr}
\hline & 1996 & 2001 \\
\hline tVT positiv & $42(16,3 \%)$ & $62(13,7 \%)$ \\
tVT Ausschluss & $215(83,7 \%)$ & $391(86,3 \%)$ \\
gesamt & $257(100,0 \%)$ & $453(100,0 \%)$ \\
\hline
\end{tabular}

(tVT: tiefe Beinvenenthrombose) 


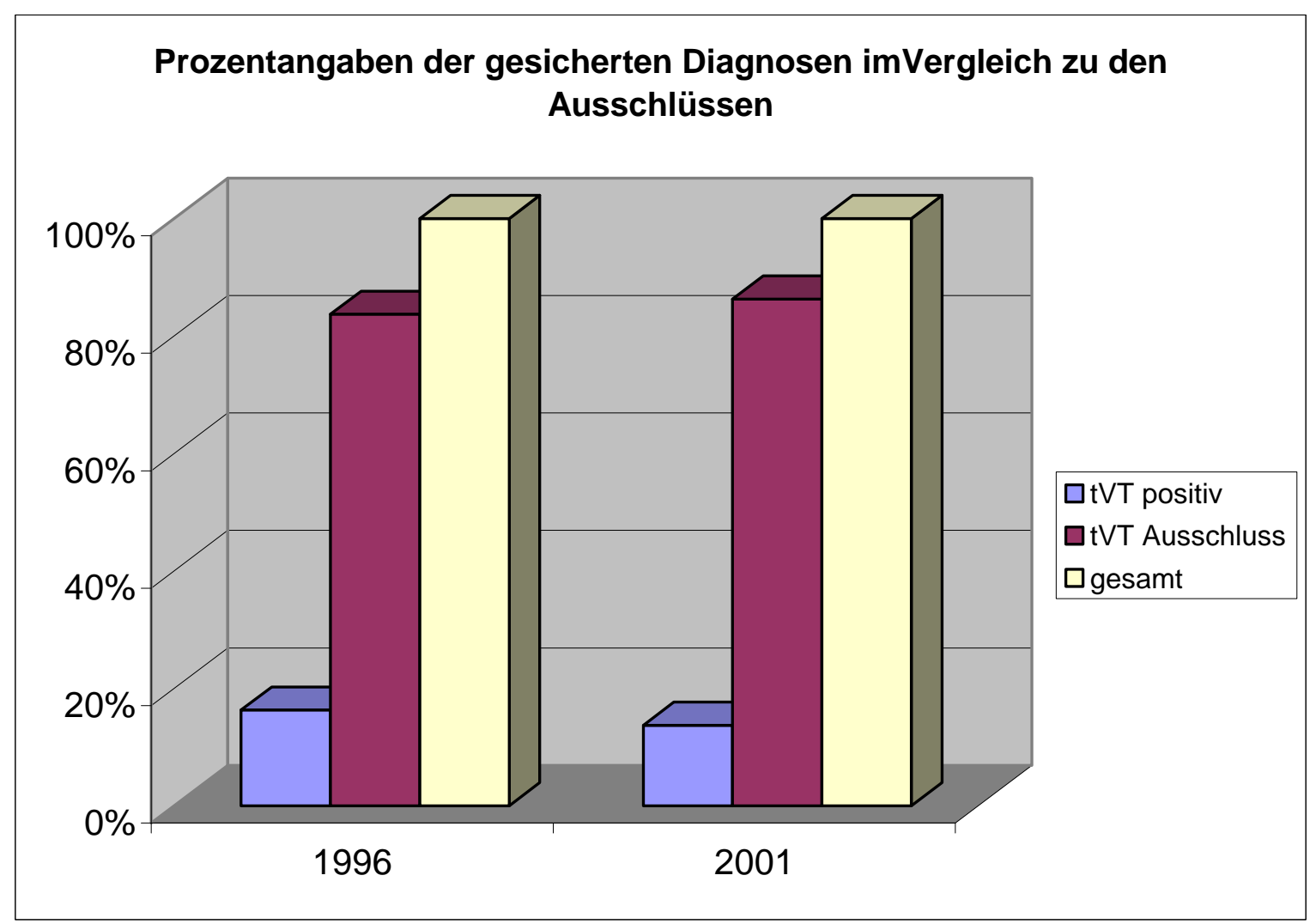

Abb.3: Diagnosen im Krankenhaus im Verhältnis zur Gesamtzahl (in \%)

Die Lokalisationsverteilung der tiefen Beinvenenthrombose ist der Tabelle 14 zu entnehmen. Die Prozentangaben beziehen sich auf die Summe aller relevanten Thrombosen in einem Jahr.

Tabelle 14: Lokalisation der tiefen Beinvenenthrombose im Krankenhaus

\begin{tabular}{lrrrr}
\hline & 1996 & 2001 \\
\hline Becken & 6 & $(14,3 \%)$ & $11 \quad(17,7 \%)$ \\
Oberschenkel & 15 & $(35,7 \%)$ & $23(37,1 \%)$ \\
Unterschenkel & $21(50,0 \%)$ & $28(45,2 \%)$ \\
\hline
\end{tabular}




\subsection{Therapie}

\subsubsection{Praxis}

Die therapeutischen Maßnahmen haben sich im Laufe der untersuchten Jahre in einigen Punkten verändert. So lässt sich in Tabelle 15 eine Verlagerung des Schwerpunktes der Therapie vom stationären in den ambulanten Bereich erkennen (1990/1 vs. $1995 / 6: \chi^{2}=6,436, p=0,011 ; 1995 / 6$ vs. $\left.2000 / 1: \chi^{2}=5,917, p=0,015\right)$.

Zudem sind ihr weitere Therapieoptionen zu entnehmen, deren individuelle Bedeutung zwischen den Doppeljahrgängen schwankte. So erfolgte 1990/1 noch bei der Hälfte der Patienten eine Lysetherapie. Diese Therapieform wurde 2000/1 bei keinem Patienten mehr angewendet. Die niedermolekularen Heparine haben deutlich an Bedeutung gewonnen. So stieg der Anteil der Patienten, die ein NMH verordnet bekamen, von 30,0\% (1990/1) über 70,6\% (1995/6) auf 95,1\% (2000/1) (1990/1 vs. 1995(6: $\chi^{2}=25,973, p=0,0145 ; 1995 / 6$ vs. $\left.2000 / 1: \chi^{2}=12,355, p=0,0004\right)$. Der Vitamin-K-Antagonist Phenprocoumon hat seinen Stellenwert in der Therapie nicht verändert.

Tabelle 15: Therapeutische Möglichkeiten und deren Stellung in der Therapie der tiefen Beinvenenthrombose (Anzahl der Einheiten absolut und in \%; Werte über 100\% möglich wegen Mehrfachnennungen; Daten der Praxis)

\begin{tabular}{|c|c|c|c|c|c|c|c|c|}
\hline \multirow[b]{2}{*}{ ambulant } & $1990 / 91$ & \multicolumn{2}{|c|}{1995} & \multicolumn{2}{|c|}{1996} & \multicolumn{2}{|c|}{2000} & 2001 \\
\hline & $3(30,0 \%)$ & 17 & $(70,8 \%)$ & 19 & $(70,4 \%)$ & 22 & $(78,6 \%)$ & 32 (97,0\%) \\
\hline stationär & $7 \quad(70,0 \%)$ & 7 & $(29,2 \%)$ & 8 & $(29,6 \%)$ & 6 & $(21,4 \%)$ & $1 \quad(3,0 \%)$ \\
\hline Lyse & $5(50,0 \%)$ & 4 & $(16,7 \%)$ & 4 & $(14,8 \%)$ & 0 & $(0,0 \%)$ & $0 \quad(0,0 \%)$ \\
\hline HEP NMH & $3(30,0 \%)$ & 18 & $(75,0 \%)$ & 18 & $(66,7 \%)$ & 26 & $(92,9 \%)$ & 32 (97,0\%) \\
\hline Phenprocoumon & $3(30,0 \%)$ & 3 & $(12,5 \%)$ & 6 & $(22,2 \%)$ & 15 & $(53,6 \%)$ & $11 \quad(33,3 \%)$ \\
\hline Kompression & $27(100,0 \%)$ & 24 & $(100,0 \%)$ & 27 & $(100,0 \%)$ & 28 & $(100,0 \%)$ & $33(100,0 \%)$ \\
\hline
\end{tabular}

(HEP NMH: niedermolekulares Heparin) 


\subsubsection{Krankenhaus}

Die Auswertung beschränkte sich auf die Unterscheidung zwischen einer ambulanten oder stationären Behandlung. Die Daten der Tabelle 16 belegen, dass auch im Krankenhaus die ambulante Therapie an Bedeutung gewonnen hat $\left(\chi^{2}=17,71\right.$, $p<0,01)$.

Tabelle 16: Therapie der tiefen Beinvenenthrombose im Krankenhaus (Angaben absolut und in \%)

\begin{tabular}{lrll}
\hline & 1996 & 2001 \\
\hline ambulant & 7 & $(16,7 \%)$ & $37(59,7 \%)$ \\
stationär & 35 & $(83,3 \%)$ & $25(40,3 \%)$ \\
\hline
\end{tabular}

Die folgende Abbildung spiegelt die Stellenwertänderung des ambulanten Therapieregimes wider. Vor allem im Krankenhaus hat sich ein Wandel zugunsten dieser Vorgehensweise vollzogen, wobei der prozentuale Anteil stationär geführter Patienten immer noch deutlich über dem der Praxis liegt.

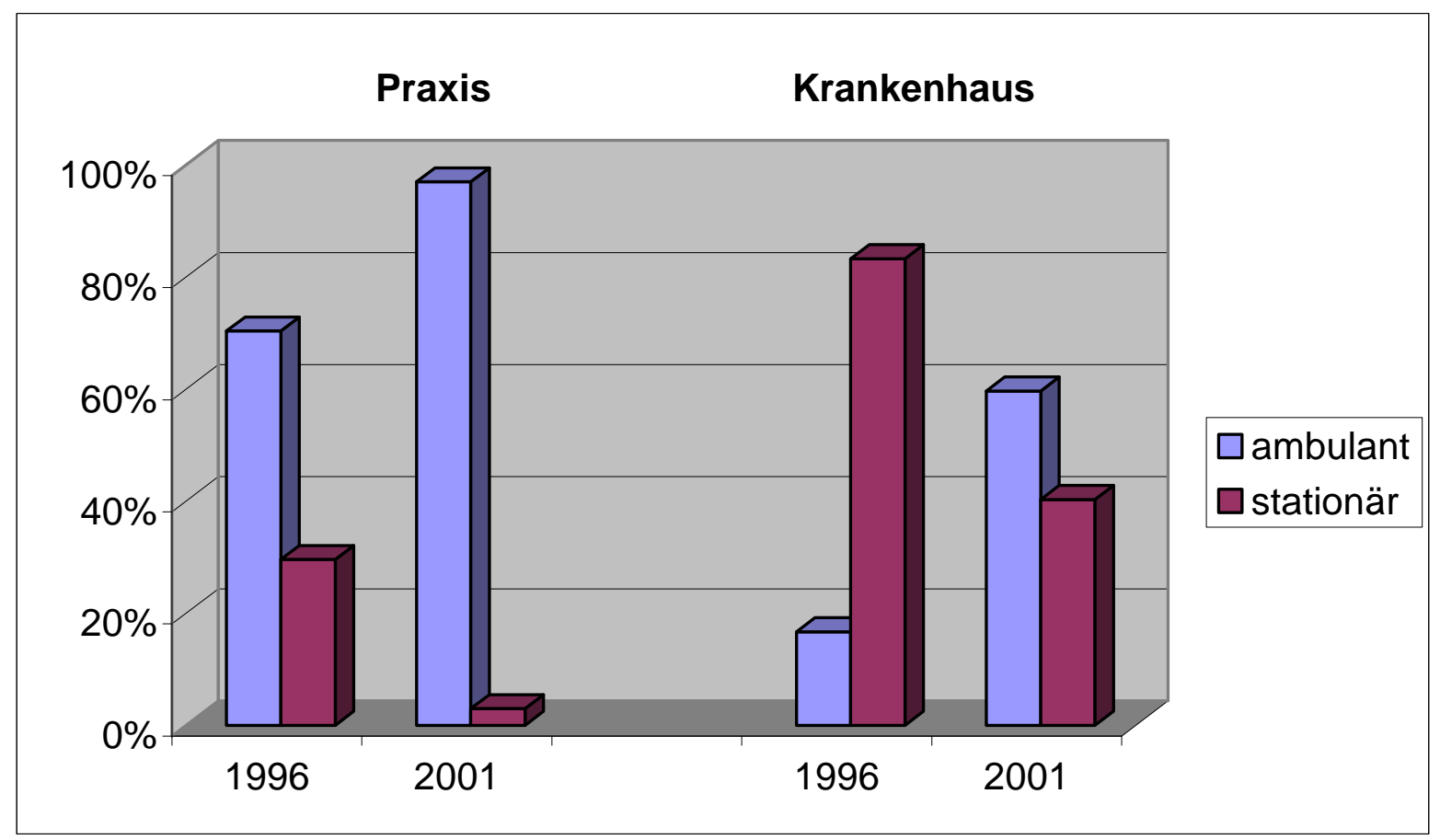

Abb.4: Vergleich des therapeutischen Vorgehens bei gesicherter tiefer Beinvenenthrombose 


\subsection{Entlassungsbrief}

In zwei Drittel der Fälle wurde schriftlich der Verlauf der Erkrankung und das weitere Procedere dokumentiert. In der Tabelle 17 sind wichtige therapeutische Maßnahmen aufgelistet. Bei keinem Patienten ließ sich die Verordnung eines unfraktionierten Heparins finden. Es wurde keine Tumordiagnostik durchgeführt. Im Verlauf der Therapie trat nie eine Blutung auf.

Tabelle 17: Prozentualer Anteil an Entlassungsbriefen im Verhältnis zum Gesamtkollektiv und deren Inhalte (in der Praxis)

\begin{tabular}{lrrrrrr}
\hline & \multicolumn{1}{c}{$1990 / 91$} & \multicolumn{2}{c}{$\mathbf{1 9 9 5 / 9 6}$} & \multicolumn{2}{c}{$\mathbf{2 0 0 0 / 0 1}$} \\
\hline Patienten gesamt & 27 & & 122 & & 222 & \\
Brief geschrieben & 18 & $(66,7 \%)$ & 74 & $(60,7 \%)$ & 154 & $(69,4 \%)$ \\
Marcumar erwähnt & 3 & $(11,1 \%)$ & 7 & $(5,7 \%)$ & 27 & $(12,2 \%)$ \\
Marcumar lebenslang & 0 & $(0,0 \%)$ & 0 & $(0,0 \%)$ & 2 & $(0,9 \%)$ \\
Heparin erwähnt & 1 & $(3,7 \%)$ & 9 & $(7,4 \%)$ & 34 & $(15,3 \%)$ \\
HEP NMH erwähnt & 1 & $(3,7 \%)$ & 6 & $(4,9 \%)$ & 33 & $(14,9 \%)$ \\
Kompression erwähnt & 10 & $(37,0 \%)$ & 32 & $(26,2 \%)$ & 77 & $(34,7 \%)$ \\
Kompression lebenslang & 1 & $(3,7 \%)$ & 2 & $(1,6 \%)$ & 4 & $(1,8 \%)$ \\
Thrombophiliediag. durchgef. & 1 & $(3,7 \%)$ & 1 & $(0,8 \%)$ & 0 & $(0,0 \%)$ \\
Verlaufskontrolle durchgef. & 20 & $(74,1 \%)$ & 73 & $(59,8 \%)$ & 111 & $(50,0 \%)$ \\
Rezidiv & 1 & $(3,7 \%)$ & 1 & $(0,8 \%)$ & 1 & $(0,5 \%)$ \\
\hline
\end{tabular}

(HEP NMH: niedermolekulares Heparin) 


\subsection{Prospektive Untersuchung}

Um die Aussagekraft der retrospektiven Erfassung zu verstärken, wurden in der Praxis prospektiv Daten erhoben. Es sollte die Frage beantwortet werden, ob bei der hohen Rate an ermittelten Ausschlussdiagnosen der Hausarzt eine sogenannte „Filterfunktion" einnimmt beziehungsweise einnehmen kann. Der Schwerpunkt lag in der Ermittlung des Aussagewertes der klinisch-körperlichen Untersuchung.

Der Tabelle 18 sind die ermittelten Patientencharakteristika zu entnehmen. Der überwiegende Teil der Patienten umschrieb die Symptome als akutes Ereignis. Wenige datierten den Beschwerdebeginn drei Monate zurück.

Tabelle 18: Patientencharakteristika bei 114 hausärztlich überwiesenen Patienten mit Verdacht auf Beinvenenthrombose (Angaben absolut und in Prozent sowie Odds ratio mit 95\%-Konfidenzintervall)

\begin{tabular}{lllll}
\hline Charakteristika & $\begin{array}{c}\text { Alle Patienten } \\
(\%)\end{array}$ & $\begin{array}{c}\text { Ohne tVT } \\
(\%)\end{array}$ & $\begin{array}{c}\text { Mit tVT } \\
(\%)\end{array}$ & $\begin{array}{c}\text { Odds Ratio } \\
{[95 \%-C l]}\end{array}$ \\
\hline Demographie & & & & \\
\hline $\begin{array}{l}\text { Alter - Jahre } \\
\quad \text { Median }\end{array}$ & 59 & 59 & 61 & n.s. \\
$\quad \begin{array}{l}\text { Spannweite } \\
\text { Geschlecht (Anzahl) } \\
\quad \text { weiblich } \\
\quad \text { männlich }\end{array}$ & $15-91$ & $15-84$ & $27-91$ & \\
\hline
\end{tabular}

\section{Vorgeschichte}

\begin{tabular}{|c|c|c|c|c|}
\hline eschwerdebeginn (Tage) & & & & \\
\hline Median & 5 & 6 & 4 & n.s. \\
\hline Spannweite & $1-90$ & $1-90$ & $1-90$ & \\
\hline anamnestisch erfragbarer Auslöser & $22(19,3)$ & $18(17,7)$ & $4 \quad(33,3)$ & n.s. \\
\hline Reise & $8(7,0)$ & $7 \quad(6,9)$ & $1 \quad(8,3)$ & \\
\hline Trauma & $6(5,3)$ & $5 \quad(4,9)$ & $1 \quad(8,3)$ & \\
\hline Immobilisation & $6(5,3)$ & $4 \quad(3,9)$ & $2(16,7)$ & \\
\hline Operation & $2(1,8)$ & $2(2,0)$ & 0 & \\
\hline Risikofaktoren ${ }^{(*)}$ & $48(42,1)$ & $41(40,2)$ & $7 \quad(58,3)$ & n.s. \\
\hline Chronisch venöse Insuffizienz & $26(22,8)$ & $22(21,6)$ & $4(33,3)$ & \\
\hline $\begin{array}{l}\text { Thromboseneigung in der Fami- } \\
\text { lien-Anamnese }\end{array}$ & $17(14,9)$ & $17(16,7)$ & 0 & \\
\hline Positive Thromboseanamnese & $16(14,0)$ & $14(13,7)$ & $2(16,7)$ & \\
\hline
\end{tabular}




\begin{tabular}{|c|c|c|c|c|}
\hline Adipositas & $8(7,0)$ & $7 \quad(6,9)$ & $1 \quad(8,3)$ & \\
\hline Schwangerschaft & $2(1,8)$ & $2 \quad(2,0)$ & 0 & \\
\hline Bekannte Thrombophilie & 0 & 0 & 0 & \\
\hline \multicolumn{5}{|l|}{ Symptome } \\
\hline Schmerzen & $100(87,7)$ & $88(86,3)$ & $12(100,0)$ & n.s. \\
\hline Schwellung & $81(71,1)$ & $70(68,6)$ & $11(91,7)$ & n.s. \\
\hline Rötung & $22(19,3)$ & $17(16,7)$ & $5 \quad(41,7)$ & $3,57[1,01-12,59]$ \\
\hline \multicolumn{5}{|l|}{ hausärztliche Versorgung } \\
\hline Kompressionsverband & $28(24,6)$ & $21(20,6)$ & $7(58,3)$ & $5,4 \quad[1,55-18,73]$ \\
\hline Kompressionsstrumpf angelegt & $18(15,8)$ & $16(15,7)$ & $2(16,7)$ & n.s. \\
\hline Antikoagulation & $20(17,5)$ & $14(13,7)$ & $6(50,0)$ & $6,29[1,78-22,26]$ \\
\hline
\end{tabular}

Körperliche Untersuchungsbefunde

\begin{tabular}{lrrrrc}
\hline Wadendruckschmerz & $45(39,5)$ & $34(33,3)$ & $11(91,7)$ & $22,0[2,72-177,53]$ \\
Umfangsdifferenz $(>1 \mathrm{~cm})$ & $72(63,2)$ & $61(59,8)$ & $11(91,7)$ & n.s. \\
$\quad$ im Knöchelbereich & $47(41,2)$ & $39(38,2)$ & $8(66,7)$ & n.s. \\
im Wadenbereich & $60(52,6)$ & $49(48,0)$ & $11(91,7)$ & $11,90[1,48-95,58]$ \\
Überwärmung & $26(22,8)$ & $19(18,6)$ & $7(58,3)$ & $6,12[1,75-21,37]$ \\
Verfärbung & $16(14,0)$ & $14(13,7)$ & $2(16,7)$ & n.s. \\
Homan-Zeichen positiv & $8(7,0)$ & $3(2,9)$ & $5(41,7)$ & $23,5[4,65-119,55]$ \\
Payr-Zeichen positiv & $8(7,0)$ & $2(2,0)$ & $6(50,0)$ & $50,0[8,27-302,45]$ \\
\hline
\end{tabular}

$\left(\mathrm{tVT}=\right.$ tiefe Venenthrombose,${ }^{()}=$Mehrfachnennungen möglich, n.s. = nicht signifikant

Ein klassischer Auslöser (z.B. längere Reise, Trauma, Immobilisation) ließ sich bei weniger als $20 \%$ der Patienten ermitteln. Knapp die Hälfte wies Risikofaktoren für Thrombosen auf.

Vom Hausarzt bereits veranlasste therapeutische Maßnahmen waren die Kompressionstherapie und die Antikoagulation. Antikoaguliert wurden 17,5\% der Patienten, davon erhielten $13 \%$ zusätzlich eine Kompressionsstrumpf bzw. Verband.

Abbildung 5 gibt in Form eines Flussdiagramms den Ablauf der Diagnostik einer tiefen Beinvenenthrombose in der Praxis wieder. Bei $28,9 \%$ der Patienten konnte die sonographische Untersuchung nicht eindeutig beurteilt werden. Ergänzend wurden D-Dimer-Tests und Phlebographien durchgeführt. In einem Fall konnte eine Muskelvenenthrombose nachgewiesen werden. Unter Einschluss der nicht sicheren Befunde ließ sich demnach ein Wert von 0,92 für die Sensitivität der Duplexsonographie errechnen. Die Phlebographie als weiterführende Diagnostik konnte bei vier Patienten das initial pathologische Untersuchungsergebnis nicht bestätigen. Somit errech- 
nete sich für die Spezifität der Sonographie ein Wert von 0,96. Der prädiktive Wert betrug positiv 0,73 , negativ 0,99 .

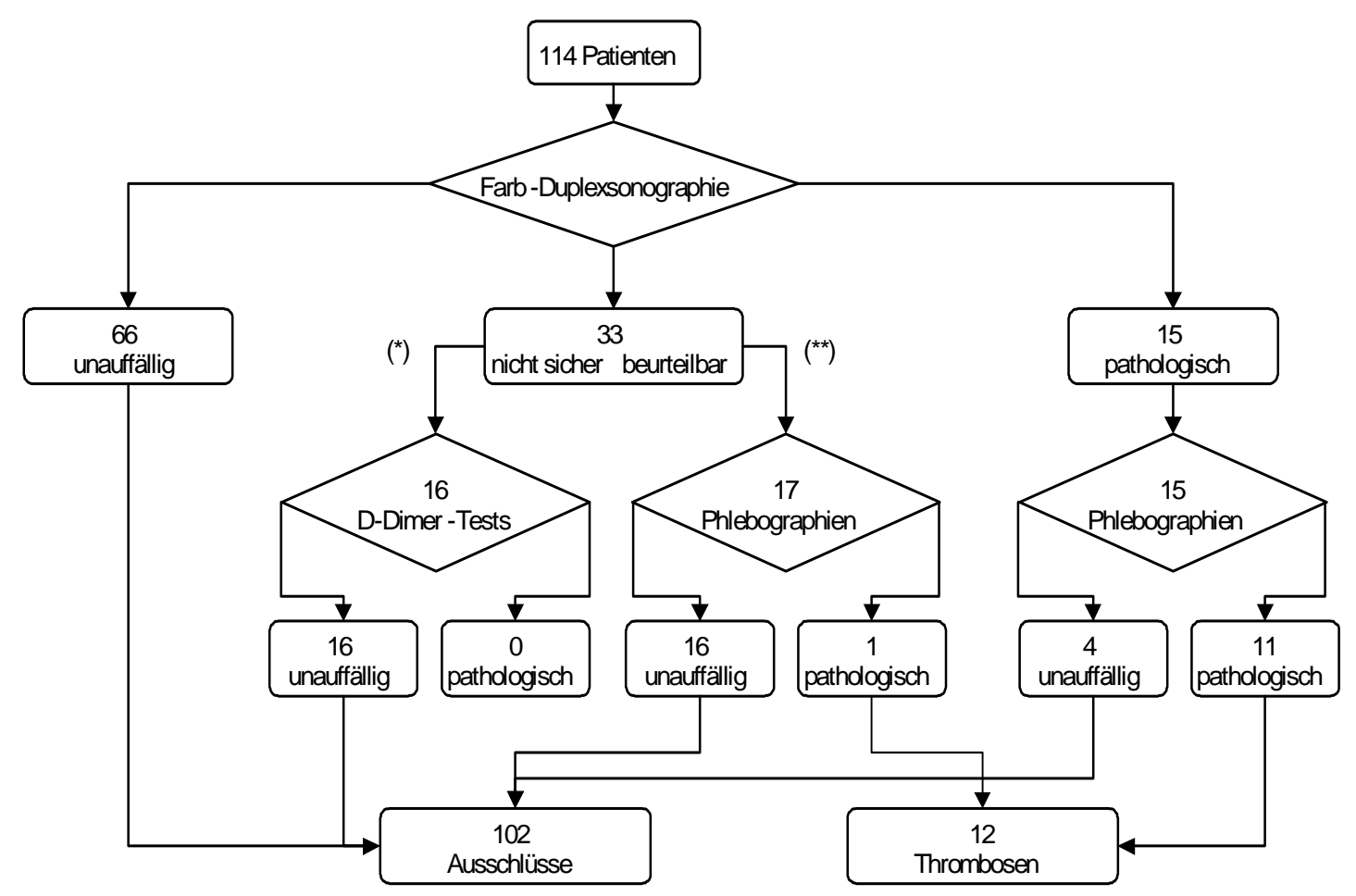

Abb. 5: Diagnostisches Vorgehen bei 114 hausärztlich überwiesenen Patienten mit Thromboseverdacht in einer phlebologischen Schwerpunktpraxis: klinische Wahrscheinlichkeit einer IVT niedrig $\left(^{(*)}\right)$, mäßig bis hoch $\left({ }^{*} *\right)$

Die Tabelle 19 verdeutlicht die Beschwerdeursachen der 102 (89,5\%) Patienten, bei denen eine tiefe Beinvenenthrombose ausgeschlossen werden konnte. Im Vordergrund standen die Varikosis und die (pseudo)radikulären Schmerzen. Eine Phlebitis war häufig der Grund der beschriebenen Symptome. Eine sichere Zuordnung der Beschwerden konnte in zwei Fällen nicht erfolgen. Der Unterpunkt der Tabelle <andere Diagnosen> beinhaltet nächtlich betonte Wadenkrämpfe ohne klinisches Korrelat. 
Tabelle 19: Diagnosen bei 114 hausärztlich überwiesenen Patienten mit Verdacht auf Beinvenenthrombose

\begin{tabular}{lr}
\hline \multicolumn{1}{c}{ Diagnosen (Anzahl) } & Anzahl (\%) \\
\hline Ausschluss Beinvenenthrombose & $102(89,5)$ \\
Stamminsuffizienz & $38(33,3)$ \\
(Pseudo-) radikuläre Beschwerden & $23(20,2)$ \\
Phlebitis & $14(12,3)$ \\
Muskuläre Beschwerden (z.B. Muskelfaserriss) & $11(9,6)$ \\
Postthrombotisches Syndrom & $4(3,5)$ \\
Lymphangitis, Erysipel & $4(3,5)$ \\
Gonarthrose & $2(1,8)$ \\
Andere & $4(3,5)$ \\
Nachweis Beinvenenthrombose & $12(10,5)$ \\
Distal & $7(6,1)$ \\
Proximal & $5(4,4)$ \\
\hline
\end{tabular}

Die Auswertung der erfassten Daten ergab deutliche Unterschiede zwischen den Patientengruppen. Die Patienten, die unter (pseudo)radikulären Schmerzen litten, wiesen signifikant weniger thrombosetypische Symptome auf als alle anderen Patienten. Hierzu zählten:

- Verfärbung: Odds ratio (OR) 0,82 (95\% Cl:0,75-0,91),

- Rötung: OR 0,76 (95\% Cl:0,68-0,85),

- Schwellung: OR 0,08 (95\% Cl:0,03-0,22),

- Umfangsdifferenz im Wadenbereich: OR 0,13 (95\% Cl:0,04-0,42) oder

- Umfangsdifferenz im Knöchelbereich: OR 0,04 (95\% Cl:0,01-0,34)

- Überwärmung: OR 0,71 (95\% Cl:0,63-0,81).

Ebenso konnten Unterschiede zwischen den Patienten mit der Diagnose einer Stammveneninsuffizienz und den restlichen Patienten festgestellt werden. Erstere beschrieben:

- häufiger Schwellungen: OR 7,23 (95\% Cl:2,04-25,66),

- seltener Schmerzen: OR 0,06 (95\% Cl:0,01-0,26) und 
- Rötungen: OR 0,71 (95\% Cl:0,62-0,82).

Unterschiede zwischen den Patientengruppen bei der Untersuchung der klassischen Zeichen einer tiefen Beinvenenthrombose traten nicht auf. Hierzu zählten die Erfassung des Homan- und Payr- Zeichen sowie der Wadendruckschmerz. Eine sichere Beurteilung und Zuordnung der Beschwerden ist nicht allein anhand dieser Untersuchungstechniken möglich. (Pseudo)radikuläre Schmerzen und die Stammveneninsuffizienz sind nicht sicher von einer tVT abgrenzbar.

Bei der körperlichen Untersuchung lies sich bei $76 \%$ der Patienten mindestens ein klinisches Zeichen der tiefen Beinvenenthrombose feststellen. Aus der Tabelle 20 gehen die Testkriterien der klinischen Thrombosezeichen hervor. Aufgrund der begrenzten Fallzahl und der niedrigen Thromboseprävalenz führte der Berechnungsversuch einer multiplen logistischen Regressionsanalyse zu keinem stabilen Modell.

Tabelle 20: Testkriterien klinischer Zeichen bei 114 hausärztlich überwiesenen Patienten mit Verdacht auf Beinvenenthrombose

\begin{tabular}{lcccc}
\hline Klinisches Zeichen & $\begin{array}{c}\text { Sensitivität } \\
{[95 \%-C l]}\end{array}$ & $\begin{array}{c}\text { Spezifität } \\
{[95 \%-C l]}\end{array}$ & $\begin{array}{c}\text { Positiv prädikti- } \\
\text { ver Wert }\end{array}$ & $\begin{array}{c}\text { Negativ prädikti- } \\
\text { ver Wert }\end{array}$ \\
\hline $\begin{array}{l}\text { Wadendruckschmerz } \\
\text { Umfangsdifferenz }(>1 \mathrm{~cm})\end{array}$ & $0,92[0,62-0,99]$ & $0,67[0,57-0,76]$ & 0,24 & 0,99 \\
$\quad$ im Knöchelbereich & $0,67[0,35-0,90]$ & $0,62[0,52-0,71]$ & 0,17 & 0,94 \\
$\quad$ im Wadenbereich & $0,92[0,62-0,99]$ & $0,52[0,42-0,62]$ & 0,18 & 0,98 \\
Überwärmung & $0,58[0,27-0,85]$ & $0,81[0,72-0,88]$ & 0,27 & 0,94 \\
Verfärbung & $0,16[0,03-0,48]$ & $0,86[0,78-0,92]$ & 0,13 & 0,90 \\
Homan-Zeichen positiv & $0,42[0,15-0,72]$ & $0,97[0,92-0,99]$ & 0,63 & 0,93 \\
Payr-Zeichen positiv & $0,50[0,21-0,78]$ & $0,98[0,93-0,99]$ & 0,75 & 0,94 \\
\hline
\end{tabular}

(95\%-Cl = 95\%-Konfidenzintervall) 


\section{Diskussion}

In der vorliegenden Arbeit wurde die Diagnostik und Therapie der tiefen Beinvenenthrombose im Zeitraum 1990 bis 2001 untersucht. In einer niedergelassenen Facharztpraxis sowie in einem regionalen Krankenhaus wurden diesbezüglich retrospektiv Daten erhoben. In der Praxis wurde ergänzend eine prospektive Untersuchung durchgeführt. Der Auswertungszeitraum umfasste die Jahre 2001 bis 2003. Die Diagnostik betreffend hat der Stellenwert der Farbduplexsonographie deutlich an Bedeutung gewonnen. Sie gilt neben der klinischen Untersuchung als das Verfahren der Wahl bei Verdacht auf eine tiefe Beinvenenthrombose. Die zusätzliche Bestimmung der D-Dimere hat im Erhebungszeitraum zugenommen. Deren Wertigkeit wird jedoch nach wie vor in der Literatur diskutiert. In beiden untersuchten Institutionen hat sich ein Wandel zur ambulanten Therapie unter Verwendung niedermolekularer Heparine vollzogen. Im Krankenhaus liegt jedoch der Anteil der stationär versorgten Patienten nach wie vor deutlich über dem der Praxis. Die Daten belegen weiterhin nicht nur eine signifikante Zunahme der tVT-Verdachtsfälle und Ausschlüsse sondern auch einen Anstieg der diagnostizierten tiefen Beinvenenthrombosen im Untersuchungszeitraum.

Die tiefe Beinvenenthrombose gehört mit einer weltweiten jährlichen Inzidenz von 1-3 Erkrankten pro 1.000 Einwohner zu den häufiger auftretenden Krankheitsbildern (Kearon 2001). Die potentiell letale Bedrohung durch eine Lungenembolie und die Gefahr der Entwicklung eines postthrombotischen Syndroms erfordern eine schnelle, sichere und effiziente Diagnostik und Therapie. Gerade in diesen Bereichen hat es in den vergangenen 10 Jahren bedeutende Veränderungen gegeben. Die bislang als "Goldstandard“ bezeichnete Phlebographie stellt nicht mehr die Untersuchungsmethode der Wahl bei Verdacht auf eine tiefe Beinvenenthrombose dar. Diesen Stellenwert nimmt die farbkodierte Duplexsonographie ein (Dietrich und Bauersachs 2002). Ausschlaggebend hierfür sind die Vorteile der nicht invasiven Ultraschalluntersuchung. Komplikationen, die bei einer phlebographischen Untersuchung auftreten können, sind ausgeschlossen. Die Ergebnisse dieser Arbeit belegen die gewachsene Bedeutung der Farbduplexsonographie in der Diagnostik. Therapeutisch ist eine ambulante Betreuung des Patienten möglich geworden. Die Etablierung der niedermolekularen Heparine und der damit verbundenen Vorteile gegenüber der Verwendung von unfraktionierten Heparinen sind hierfür ursächlich. In der vorliegenden 
Studie wird neben einer kritischen Betrachtung dieser neuen Möglichkeiten, deren Anwendung und damit Umsetzung anhand von zwei Institutionen überprüft. Es soll beurteilt werden, ob auf Grund der neuen Erkenntnisse eine sichere primärärztliche Versorgung durch den Hausarzt bei Verdacht auf eine tVT durchführbar ist.

\subsection{Körperliche Symptome}

Klinische Symptome sowie verschiedene Untersuchungszeichen sind nicht allein zur sicheren diagnostischen Abklärung eines Patienten ausreichend. Sie können lediglich in Kombination mit anderen apparativen Verfahren die Wahrscheinlichkeit für eine Erkrankung erhöhen beziehungsweise erniedrigen. Vor allem die prospektive Untersuchung konnte zeigen, dass auch bei einer bereits vorselektierten Gruppe von Patienten mittels der klinisch-körperlichen Untersuchung keine sichere Diagnostik durchgeführt werden kann. Somit ist es dem Hausarzt nicht möglich ohne die Verwendung weiterer Verfahren zuverlässig eine IVT zu bestätigen oder diese auszuschließen. Andere Autoren kommen zu dem gleichen Ergebnis. So wird beschrieben, dass die klinische Symptomatik einer akuten tiefen Beinvenenthrombose ein breites Feld von völliger Symptomfreiheit bis hin zu starken Schmerzen und massiven Schwellungen aufzeigen kann. Eindeutige Diagnosen können nur unter Einsatz weiterer diagnostischer Mittel gestellt werden (Kearon et al. 1998). Die Ergebnisse der retrospektiven als auch der prospektiven Untersuchung bestätigen die anderer Studien. Laut einer Arbeit von Aschwanden et al. (1999), bei der 343 Patienten mit Verdacht auf eine tiefe Beinvenenthrombose untersucht wurden, liegt die Sensitivität der Inspektion, Palpation und die Überprüfung der Beinumfänge zwischen $67 \%$ und $84 \%$. Für die Spezifität errechnete sich ein Wert von $51 \%$ bis $56 \%$. Die Schwankungen ergeben sich dabei aus der unterschiedlichen Lokalisation der tVT. Vor allem die sichere Diagnostik einer Unterschenkelvenenthrombose ist schwierig. In einer Untersuchung von Perone et al. (2001) stellte sich heraus, dass allein klinisch keine eindeutige Aussage getroffen werden kann. Das Ergebnis der weiterführenden Diagnostik war häufig ein Normalbefund bei klinisch vermuteter tVT. Die Autoren mehrerer Studien haben sich dementsprechend in den letzten Jahren damit beschäftigt, die Aussagekraft der klinisch körperlichen Untersuchung in Verbindung mit anderen Verfahren zu bewerten. Dabei standen vor allem die Bestimmung des D-Dimer-Spiegels und die farbkodierte Duplexsonographie im Vordergrund. 


\subsection{Farbduplexsonographie versus Phlebographie}

Die Weiterentwicklung der apparativen Diagnostik führte zu einer Stellenwertänderung der farbkodierten Duplexsonographie (FKDS) und der aszendierenden Beinvenenphlebographie. Hierbei wird das zuletzt genannte etablierte Verfahren immer stärker in den Hintergrund gedrängt. In einer aktuellen Studie bei der die Jahre von 1979 bis 1999 erfasst wurden, konnte beispielhaft neben einer deutlichen Abnahme der durchgeführten Phlebographien eine generelle Zunahme der stattgehabten Ultrasonographieuntersuchungen festgestellt werden (Stein et al. 2004). Dabei ist die diagnostische Sicherheit der FKDS vor allem bei symptomatischen Patienten im Vergleich zur Standardmethode der Phlebographie als hoch zu bewerten (Kearon et al 1998). Laut einer Übersichtsarbeit von Dietrich und Bauersachs (2002) erreicht die FKDS eine Sensitivität von $62 \%$ bis $97 \%$. Die Schwankungen resultieren aus der deutlichen Untersucherabhängigkeit des Verfahrens (Dietrich und Bauersachs 2002). Eine Studie zeigte zudem eine hohe Rate an nicht sicher beurteilbaren Duplexsonographien im Bereich des Unterschenkels (Noren et al. 2002). Das Vorgehen in der Praxis beinhaltete in solchen Fällen eine sich anschließende phlebographische Abklärung. Im Vergleich der beiden Verfahren zeigt die FKDS ausgeprägte Vorteile, die sich aus der "Nichtinvasivität“ dieser Untersuchungsmethode ergeben. Die Anwendung kann beliebig oft wiederholt werden und bietet die Möglichkeit thrombotische Verschlüsse ohne Strahlenbelastung nachweisen zu können (Rudofsky und Michler 2002). Sie wird als eine preiswerte und für den Patienten nicht belastende Methode angesehen, die flexibel und somit auch am Krankenbett einsetzbar ist (Kearon et al. 2001). Die Phlebographie wird hingegen als ein invasives und risikoreiches Verfahren beschrieben, welches einen hohen personellen und technischen Aufwand erfordert (Weber 1998, Oudega et al. 2005 b). Neben der Strahlenbelastung für den Patienten sind allergische Reaktionen auf das Kontrastmittel sowie Nierenschädigungen möglich. Es kann zur Induktion weiterer Thrombosen und oberflächlicher Phlebitiden kommen (Weber 1998). Ebenso ist die Aussagekraft der Phlebographie begrenzt (Tatò 2002, Oudega et al. 2005 b). Die diagnostische Sicherheit liegt laut einiger Studien bei $90 \%$ bis $100 \%$ (Miller et al. 1996, Weber 1998). In einem relevanten Teil der Fälle ist nicht das gesamte Venensystem darstellbar und somit keine sichere Befundung möglich (Rudofsky und Michler 2002). Die häufig nicht darstellbare Gruppe der Venae-tibialis-anteriores ist hingegen mit hoher Treffsicherheit mit der Duplexsonographie beurteilbar (Bjorgell et al. 2000). Ein weiterer Nachteil der Phlebographie ist die eingeschränkte Durchführbarkeit der Untersuchung. Die nicht mögliche Punk- 
tion der Fußrückenvene, eine bestehende Schwangerschaft, eine diagnostizierte Hyperthyreose oder Phlegmasia coerulea dolens sowie eine Kontrastmittelallergie stellen Kontraindikationen dar (Diehm et al. 1997). Umstände, die die Durchführbarkeit einer Ultraschalluntersuchung erschweren oder unmöglich machen sind laut Literatur starke Adipositas, ein massives Lymphödem sowie postthrombotische Veränderungen (Rudofsky und Michler 2002). Daraus ergeben sich Unterschiede für die Indikationsstellung und allgemeine Verwendung der Farbduplexsonographie. Einige Autoren kommen zu dem Ergebnis, dass die Ultraschalluntersuchung die Methode der Wahl bei Thromboseverdacht darstellt (Dietrich und Bauersachs 2002). Andere wiederum empfehlen auf Grund der erschwerten Bedingungen im Bereich des Unterschenkels eine Verwendung nur oberhalb der Vena poplitea. Im Wadenbereich sollte zur sicheren Diagnostik die Phlebographie benutzt werden (Baarslag et al. 2002). Der frühere Goldstandard wird nach wie vor als Methode der Wahl bei fraglichen Befunden in der FKDS angesehen (Baarslag et al. 2002). Letztere Aussage denkt sich dabei mit der Vorgehensweise in der phlebologischen Schwerpunktpraxis. In der vorliegenden Untersuchung konnte gezeigt werden, dass der Einsatz der farbkodierten Duplexsonographie zugenommen hat. Wurde in den Jahren 1990/1 nur bei rund 70\% der relevanten Praxispatienten diese Methode zur diagnostischen Abklärung verwendet, so stieg deren Einsatz auf 100\% in den Jahren 2002/3. Bis zum Jahr 2001 nahm dagegen der prozentuale Anteil der durchgeführten Beinvenenphlebographien um ca. $50 \%$ ab. Auch die Daten des Krankenhauses spiegeln den gewachsenen Stellenwert der FKDS wieder. Der bei jedem Patienten mit Verdacht auf eine tiefe Beinvenenthrombose durchgeführten FKDS stehen für den Zeitraum 1996 nur zwei Phlebographien $(0,8 \%)$ gegenüber. An dieser Stelle ist zu bemerken, dass sich der sehr niedrige Anteil an stattgehabten Phlebographien nicht zuletzt aus der Kompetenz des verantwortlichen Arztes im Bezug auf die Befundung der durchgeführten Duplexsonographien erklärt (Ausbilder der deutschen Gesellschaft für Ultraschallmedizin).

In der niedergelassenen Facharztpraxis und im Krankenhaus hat ein Wandel in der Diagnosesicherung stattgefunden. Die FKDS ist ein etabliertes nicht invasives Verfahren, mit dem in geübter Hand sicher, schnell und kostengünstig eine Diagnose gestellt werden kann. Die Phlebographie nimmt nicht mehr den Stellenwert des sogenannten "Goldstandards" ein. Sie wird überwiegend bei unsicheren Befunden und gleichzeitig hoher klinischer Wahrscheinlichkeit für eine tVT durchgeführt. 


\subsection{Der Stellenwert der D-Dimer-Testung}

Der Nachweis der bei der Fibrinolyse entstehenden D-Dimere erlangt in der Diagnostik der tiefen Beinvenenthrombose immer mehr an Bedeutung. Die Ergebnisse zahlreicher Studien konnten eine hohe Sensitivität für die tiefe Beinvenenthrombose nachweisen, die je nach Testverfahren 85 bis $98 \%$ beträgt (Aschwanden et al. 1999, Perrier und Bounameaux 2001). Es ließ sich feststellen, dass die D-Dimere bereits einige Tage vor der Entstehung einer IVT einen relevanten Anstieg zeigen können (Grohmann 1999). Untersuchungen ergaben eine Korrelation der Höhe der D-DimerKonzentration mit der Ausdehnung der Thrombose, sowie eine umgekehrte Korrelation mit dem Alter der Thrombose (Knecht und Heinrich 1995). Unter Berücksichtigung der Halbwertszeit der D-Dimere im Plasma von 8 Stunden (Bradley et al. 2000) und des Normbereiches von $0,5 \mathrm{mg}$ Fibrinogenäquivalent/Liter, lässt sich eine Aussage bezüglich einer tVT-Wahrscheinlichkeit treffen (Tatò 2002). Allerdings muss die in mehreren Studien herausgearbeitete geringe Spezifität bei hoher Sensitivität dieser Methode berücksichtigt werden (Kearon et al. 2001, Kelly et al. 2002, Tatò 2002). Sie erklärt sich aus der Erhöhung der D-Dimer-Menge als Folge einer aus verschiedenen Gründen gesteigerten Gerinnungsaktivität (konsumierende Erkrankungen, Sepsis, postoperativ und posttraumatisch) (Le Gal et al. 2006). Es konnte bei Gesunden eine Geschlechts- und Altersabhängigkeit ermittelt werden. Eine Erhöhung bei Schwangeren und Rauchern wurde beobachtet (Dempfle et al. 1996). In einer Studie von Knecht und Heinrich (1997) ergab die Untersuchung von insgesamt 156 Patienten Werte von $98 \%$ für die Sensitivität beziehungsweise $44 \%$ für die Spezifität. Die Ergebnisse von Wheeler und Anderson (1995) (99\% bzw. 53\%) deckten sich hiermit. Sie schlussfolgerten, dass mittels einer normwertigen D-Dimer-Konzentration eine tVT sicher ausgeschlossen werden kann. Bei erhöhten Werten sollten weitere Maßnahmen zur Diagnosesicherung durchgeführt werden. Andere Autoren teilen diese Meinung. Sie kommen zu dem Ergebnis, dass ein nicht erhöhter D-Dimer-Wert eine akute tVT mit hoher Sicherheit ausschließt (Aschwanden et al. 1999, Legnani et al. 1999, de Moerloose 2000, Wells et al. 2000, Perrier und Bounameaux 2001, Rathbun et al. 2004, Oudega et al. 2005 c). Vor allem in Verbindung mit der klinischen Vortestwahrscheinlichkeit, stellt die Messung der D-Dimere ein gutes Screening-Verfahren bei ambulanten Patienten dar (Tatò 2002, Oudega et al. 2005 c). In einer aktuellen Untersuchung konnte festgestellt werden, dass ein negativer SimpliRed-Test in Verbindung mit einer geringen klinischen Wahrscheinlichkeit zum sicheren Ausschluss einer tiefen Beinvenenthrombose ausreicht. Diese Arbeit umfasst die 
Ergebnisse mehrerer Studien aus dem Zeitraum 1993 bis 2003 und schließt somit über 5000 Patienten in die Auswertung ein. Die Inzidenz der Erkrankung lag in dieser Kombination bei lediglich $0,5 \%$. Eine weitere Reduktion auf $0,4 \%$ ließe sich unter Verwendung eines hoch sensitiven D-Dimer-Testes erreichen (Fancher et al. 2004).

Die vorliegende Studie zeigt, dass die Bestimmung der D-Dimer-Konzentration einen über den Zeitraum gewachsenen Stellenwert in der Diagnostik der IVT einnimmt. In Übereinstimmung mit den Ergebnissen anderer Arbeiten konnte die Auswertung der Praxisdaten zeigen, dass diese Untersuchungsmethode in den letzten Jahren vermehrt angewendet wurde. Vor allem die prospektiv erhobenen Daten verdeutlichen, dass das Vorgehen in der Diagnostik einer IVT die Bestimmung der D-Dimere beinhaltet. Dieser wird bei allen Patienten bestimmt, bei denen die FKDS eine geringe tVT-Wahrscheinlichkeit zum Ergebnis hat. Eine Phlebographie wird nur in den Fällen durchgeführt bei denen die FKDS nicht sicher beurteilt werden kann. Dieses Vorgehen deckt sich mit den Erkenntnissen anderer Studien, die die Aussagekraft der DDimer-Messung ebenso stark einschränken (Kearon et al. 2001, Kelly et al. 2002, Tatò 2002). Ein entscheidender Fortschritt würde sich aber nur dann einstellen, wenn mittels der D-Dimer-Bestimmung auf weitere apparative Maßnahmen verzichtet werden könnte. So erreichte die Kombination aus hoher klinischer Wahrscheinlichkeit und pathologisch erhöhtem D-Dimer-Werten in einer Studie eine Sensitivität von $100 \%$ (Ilkhanipour et al. 2004). Hier wurde die Vermutung geäußert, dass gegebenenfalls bei dieser Befundkonstellation auf weitere Maßnahmen verzichtet werden kann. Die mangelnde Spezifität bei gleichzeitig hoher Sensitivität des Verfahrens erlaubt jedoch laut anderer Autoren nur eine sichere Ausschlussdiagnostik (Kearon et al. 2001, Tatò 2002, Kelly et al. 2002, Tovey und Wyatt 2003). Zusammen mit dem Ergebnis der klinischen Untersuchung wäre dies eine mögliche Option für einen Thromboseausschluss in der Hausarztpraxis. Nur pathologische D-DimerKonzentrationen in Verbindung mit einer klinischen Symptomatik würden dann eine weiterführende Diagnostik erfordern. Unter diesen Voraussetzungen würden sich Einsparungen für das Gesundheitssystem ergeben. Die Anzahl nötiger Überweisungen und damit verbundener weiterer Untersuchungsverfahren ließe sich deutlich reduzieren (Aschwanden et al. 1999). Beispielhaft wäre die Durchführung einer invasiven Phlebographie erheblich seltener nötig (Knecht 1994). Die Praxisdaten bestätigen diesen Wandel. Bei ca. 50\% der Patienten musste nicht wie bislang eine phle- 
bographische Untersuchung zur genaueren Abklärung hinzugefügt werden. In der Hälfte der Fälle konnte somit auf das invasive und aufwendige Verfahren verzichtet werden.

\subsection{Niedermolekulare Heparine versus unfraktionierte Heparine}

Die Entwicklung der niedermolekularen Heparine führte zu Veränderungen in der Prophylaxe und Therapie der tiefen Beinvenenthrombose. Dabei konnten die unerwünschten Effekte, der seit 50 Jahren verwendeten unfraktionierten Heparine deutlich reduziert werden (Stammler und Diehm 1998). Die pharmakodynamischen und pharmakokinetischen Unterschiede zwischen den beiden Medikamentengruppen trugen hierzu wesentlich bei. Die Bioverfügbarkeit der niedermolekularen Heparine liegt bei subkutaner Applikation bei 90-100\% (Hausman 1999). Unfraktionierte Heparine wirken bei gleicher Gabe nur zu einem Viertel (Prandoni et al. 2004). Die veränderte Eliminationskinetik der niedermolekularen Heparine bedingt eine verlängerte Halbwertszeit und ermöglicht so eine gewichtsadjustierte Dosierung. Eine geringe Anzahl an Injektionen ist erforderlich (Diehm et al. 2002). Der dosisabhängige antikoagulatorische Effekt lässt sich im Gegensatz zu den unfraktionierten Heparinen besser vorhersagen. Bei einer großen therapeutischen Breite weisen sie ein deutlich günstigeres Nebenwirkungsprofil auf (Hirsh et al. 1998). Diese Form der Medikation bietet im primärärztlichen Bereich die Möglichkeit eine IVT sicher ambulant betreuen und therapieren zu können. Grundvoraussetzung ist die Kontrolle der Thrombozytenwerte sowie der Ausschluss von Kontraindikationen. Eine bestehende Niereninsuffizienz würde zum Beispiel zu einer Anreicherung des Wirkstoffes im Blut führen. Der gerinnungshemmende Effekt wäre zu ausgeprägt. Das Restrisiko für die Entstehung einer Heparin-induzierten-Thrombozytopenie (HIT) liegt bei ca. 0,2\% (Warkentin 2002). Ob regelmäßige Laborkontrollen des Blutbildes erfolgen müssen, ist derzeit nicht abschließend geklärt. Einige Autoren vertreten die Meinung, dass diese nicht immer durchzuführen seien (Tovey und Wyatt 2003). Zur Sicherheit solle für spätere Vergleiche ein Ausgangswert der Thrombozyten bestimmt werden. Nötige Blutplättchenzahlbestimmungen wären bei fortgesetzter Heparintherapie am 5. und 10. Tag durchzuführen (Warkentin 2002). Eine andere Vorgehensweise würde die erstmalige Kontrolle am 3. Therapietag beinhalten und in der Folge an jedem 2. Tag (Hyers et al. 2001). Eine solche Versorgung ist regelhaft beim Hausarzt möglich. In Kombinati- 
on mit den Erkenntnissen in der Diagnostik kann er bei einem großen Teil der Erkrankten sicher die gesamte Betreuung übernehmen.

Studien wiederlegen die ursprüngliche Annahme, dass nur durch die intravenöse Gabe eines unfraktionierten Heparins eine adäquate Therapie erfolgen kann. Die initiale Heparinisierung ist mittels NMH möglich (Holmstrom et al. 1997, Merli et al. 2001, Prandoni et al. 2004). Dies ist unabhängig von der Tatsache, dass nur unter Verwendung unfraktionierter Heparine eine sofortige Antikoagulation möglich ist (Schellong und Schwarz 2002). Eine Metaanalyse über 11 randomisierte Studien konnte eine Gleichwertigkeit beider Medikamentengruppen belegen, wobei deutlich weniger größere Blutungen unter Verwendung von $\mathrm{NMH}$ auftraten (Dolovich et al. 2000). Die Inzidenz unerwünschter Nebenwirkungen ist deutlich geringer als bei den unfraktionierten Heparinen (Diehm et al. 2002). Die Daten der vorliegenden Untersuchung belegen den über den Beobachtungszeitraum gestiegenen Einsatz der niedermolekularen Heparine. Bis auf einen Erkrankungsfall wurden im Jahr 2001 alle Patienten mit einem fraktionierten Heparin behandelt und ambulant geführt. Anfang der neunziger Jahre betrug deren Anteil lediglich 30\%. Wie in einer Studie von Spandorfer et al. (1999) gezeigt werden konnte, bevorzugen auch die behandelten Patienten diese Form der medikamentösen Therapie. Als Gründe wurden die im Vergleich verbesserte Mobilität und Lebensqualität angegeben. Nur bei bestehender Niereninsuffizienz sollten unfraktionierte Heparine verwendet werden. Die Wirkung der NMH kann nicht an der PTT abgelesen werden. Der labortechnisch aufwendig zu bestimmende Anti-Xa-Spiegel ist erforderlich, da sonst eine potenziell gefährliche Kumulation der NMH droht (Schellong und Schwarz 2002). Die Dosisanpassung wird als kompliziert beschrieben.

Die Bewertung des Cochrane Review ergab eine bessere Effektivität und Sicherheit der fraktionierten Heparine (van den Belt et al. 2004). Mehrere Autoren empfehlen basierend auf dieser Datenlage den Einsatz der NMH beim Krankheitsbild der tiefen Beinvenenthrombose (Aschwanden et al. 2001, Schraibman et al. 2004, van den Belt et al. 2004). Sie stellen die erste Wahl in der Behandlung dar (Botella und Gomez 2004). Auch bei einer der akuten Lungenembolie ist die bessere Wirksamkeit und der komplikationsärmere Verlauf nachgewiesen worden (Alonso et al. 2005).

Die Ergebnisse der vorliegenden Studie belegen den Wandel in der medikamentösen Therapie der tiefen Beinvenenthrombose. Die niedermolekularen Heparine werden 
unter Berücksichtigung der Kontraindikationen routinemäßig in der alltäglichen Praxis angewendet. Sie bieten die Möglichkeit einer effektiven und sicheren Behandlung im primärärztlichen Bereich. Der Hausarzt kann in einem großen Teil der Erkrankungsfälle die vollständige Betreuung der Patienten übernehmen.

\subsection{Ambulante Behandlung einer tiefen Beinvenenthrombose}

Bereits vor den beiden richtungsweisenden Studien aus Kanada und Europa befasste sich der Internist Blättler (1991) mit der ambulanten Therapieführung eines an einer tiefen Beinvenenthrombose erkrankten Patienten. Er stützte sich auf die Tatsache, dass der Zusammenhang zwischen der Bewegung des Körpers und der Entstehung einer Embolie bislang in keiner Studie belegt worden war. Erst spätere Arbeiten konnten die Annahme bestätigen, dass auf die bislang praktizierte Immobilisation des Patienten in einem großen Teil der Fälle verzichtet werden kann (Koopman et al. 1996, Levine et al. 1996). Die Studien hatten eine ambulante Therapieführung unter Verwendung niedermolekularer Heparine untersucht. Als Vergleich diente eine konventionelle Vorgehensweise mit unfraktionierten Heparinen. Es wurden keine signifikanten Unterschiede bezüglich Thromboserezidiven, starken Blutungen und Sterblichkeit festgestellt. Die Ergebnisse ließen sich mit der retrospektiven Auswertung der Praxisdaten bestätigen. Einige Autoren nahmen auf Grund dessen Abstand von der sonst üblichen strikten Bettruhe des Patienten (Schellong et al. 1999, Aschwanden et al. 2001, Partsch 2001). Partsch (2001) konnte nachweisen, dass sich die Inzidenz für neue szinthigraphisch darstellbare Lungenembolien im Vergleich zu einer immobilisierten Patientenkontrollgruppe nicht erhöht.

Die Vorteile der niedermolekularen Heparine eröffneten die Möglichkeit einer ambulanten Therapie. Laut Studienlage gilt sie als genauso sicher und effektiv wie die stationäre Vorgehensweise (Aschwanden et al. 2001, Schraibman et al. 2004). Sie ist gut in die Praxis umsetzbar und nur mit wenigen Komplikationen verbunden (Offermann et al. 2001, Schwartz et al. 2001). Eine einmal tägliche Injektion eines niedermolekularen Heparins ist möglich. Zusammenfassend ist diese Form der Medikation für die Patienten sehr gut durchführbar (Stammler und Diehm 1998). Kostenanalysen zeigen, dass eine Heimtherapie mit niedermolekularen Heparinen zu erheblichen Einsparungen im Gesundheitssystem führt (Boccalon et al. 2000).

Der aktuelle Cochrane-Review hat zum Ergebnis, dass in den angelsächsischen Ländern bei fast jedem Patienten eine ambulante Therapie durchgeführt wird. Stu- 
dien, die die ambulante mit der stationären Behandlung vergleichen, sind daher zukünftig nicht mehr umsetzbar (Schraibman et al. 2004).

Die vorliegende Arbeit konnte dieses Ergebnis zum Teil bestätigen. Über den Beobachtungszeitraum hat der Anteil an ambulant geführten Patienten kontinuierlich zugenommen. Anfang der 90er Jahre wurden in der Praxis 30\% der Patienten mobilisiert und mit niedermolekularen Heparinen versorgt. Dieser Anteil steigerte sich fortlaufend und erreichte im Jahr 2001 einen Wert von 97\%. Auffällig ist jedoch, dass diese Entwicklung im Krankenhaus langsamer vonstatten ging. Hier wurden 1996 noch rund $83 \%$ der Erkrankten stationär behandelt. Im gleichen Zeitraum waren es in der Praxis nur rund 29\%. Den Daten lässt sich entnehmen, dass die Patientenverteilung auf die beiden untersuchten Institutionen nicht gleichmäßig erfolgte. Im Vergleich liegt der Anteil an Beckenvenenthrombosen im Krankenhaus deutlich höher als in der Praxis. Daraus könnte sich zumindest teilweise die im Krankenhaus hohe Rate an dokumentierten stationären Behandlungen im Jahr 2001 erklären. Studien belegen jedoch, dass die ambulante Therapie der tiefen Beinvenenthrombose unabhängig von deren Lokalisation und Größe erfolgen kann (Partsch und Blättler 2000). Auch die Leitlinie der deutschen Gesellschaft für Phlebologie kommt zu dem Ergebnis, dass therapeutisch bei einer akuten tiefen Beinvenenthrombose sowohl ein ambulantes als auch ein stationäres Vorgehen durchführbar ist. Dabei stellen die Größe und Art der Thrombose keine Entscheidungskriterien dar (Blättler et al. 2003). Wells et al. (1998) kamen anhand ihrer Untersuchungen zu dem Ergebnis, dass 80\% der Patienten, die sich ambulant mit einer VVT vorstellen, gut und sicher zu Hause behandelt werden können. Wichtige Vorraussetzungen seien die definitive Sicherung der Diagnose mittels eines bildgebenden Verfahren, die Klärung der Thrombusursache, eine effektive Kompressionstherapie, der Ausschluss einer symptomatischen Lungenembolie, eine gute Compliance des Patienten und eine gute Erreichbarkeit des jeweiligen Krankenhauses (Diehm et al. 2002). Indikationen, die neben diesen zu erfüllenden Kriterien eher für eine stationäre Behandlung des Patienten sprechen, sind eine bestehende Immobilisation beziehungsweise Pflegebedürftigkeit und eine damit verbundene nicht adäquate häusliche Betreuung. Weiterhin postoperative Zustände, diagnostizierte Tumorleiden sowie ein erhöhtes Blutungsrisiko (Schellong und Schwarz 2002).

Die vorliegende Arbeit belegt, dass ein erheblicher Anteil der Patienten ambulant betreut werden kann. Dies steht im Einklang mit den Ergebnissen der bereits angeführ- 
ten Studien. Die häusliche Therapie gilt als genauso sicher und wirksam wie eine stationäre Vorgehensweise (Schraibman et al. 2004). Schwarz et al. (2001) konnten zeigen, dass die Mortalität der hospitalisierten Patienten signifikant höher liegt als die der ambulant betreuten Patienten. Sie berücksichtigten in ihrer Untersuchung 202 Erkrankungsfälle. Vor allem in der untersuchten Praxis konnte eine deutliche Steigerung festgestellt werden. Hier wurde fast jeder Patient ambulant betreut. Warum im Krankenhaus die Ergebnisse zahlreicher wissenschaftlicher Arbeiten noch nicht in diesem Maße umgesetzt wurden, bleibt offen. Hier ließ sich für den Zeitraum 2001 noch ein stationärer Behandlungsanteil von $40 \%$ ermitteln.

Eine mit einer ambulanten Therapieführung einhergehende deutliche Kostenreduktion für das Gesundheitssystem wäre eine wichtige Begleiterscheinung. Ihr kommt gerade in der heutigen Zeit eine gewachsene Bedeutung zu (Koopman et al. 1996).

\subsection{Zunahme der tVT-Verdachtsfälle}

Auffällig in unserer Untersuchung war die Zunahme der Verdachtsfälle sowohl in der Praxis als auch im Krankenhaus. Ursprünglich hatten wir angenommen, dass es zu einer Verschiebung der Patientenzahlen zwischen den beiden Institutionen gekommen ist. Dabei vermuteten wir bei einer Zunahme in der Praxis eine gleichzeitige Abnahme der Fallzahlen im Krankenhaus. Die Ursache für die generelle Steigerung der möglichen Erkrankungsfälle könnte in einer größeren Sensibilisierung der behandelnden Ärzte und der Bevölkerung für die Thematik VVT liegen. So wurde in den letzten Jahren zunehmend über einzelne Erkrankungsfälle in den Medien berichtet, die meist im Zusammenhang mit einer längeren Reise auftraten. Angesehene Tageszeitungen, wie die „Frankfurter Allgemeine Zeitung“, das „Hamburger Abendblatt“ oder die „Welt“ informierten die Bevölkerung mit einer steigenden Anzahl von Artikeln über die sogenannte „Reise“- beziehungsweise „Flugthrombose“. Es ließ sich eine leichte Zunahme der Berichterstattungen zu diesem Thema in medizinischen Fachzeitschriften feststellen. Die folgenden Abbildungen verdeutlichen diese Entwicklung. 


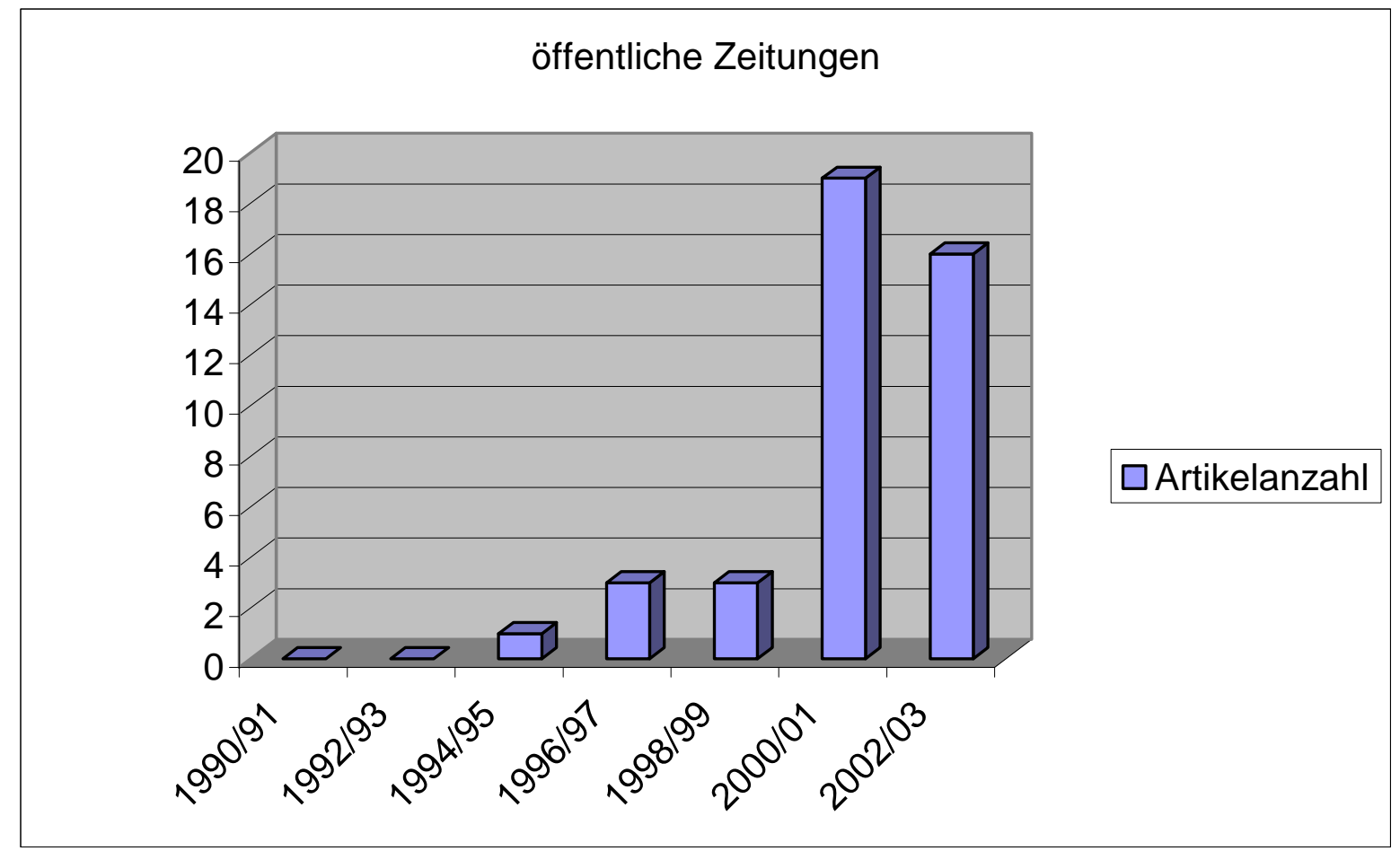

Abb. 6: Entwicklung der Artikelanzahl in öffentlichen Zeitungen (FAZ, DIE WELT, Hamburger Abendblatt) zum Thema tiefe Beinvenenthrombose.

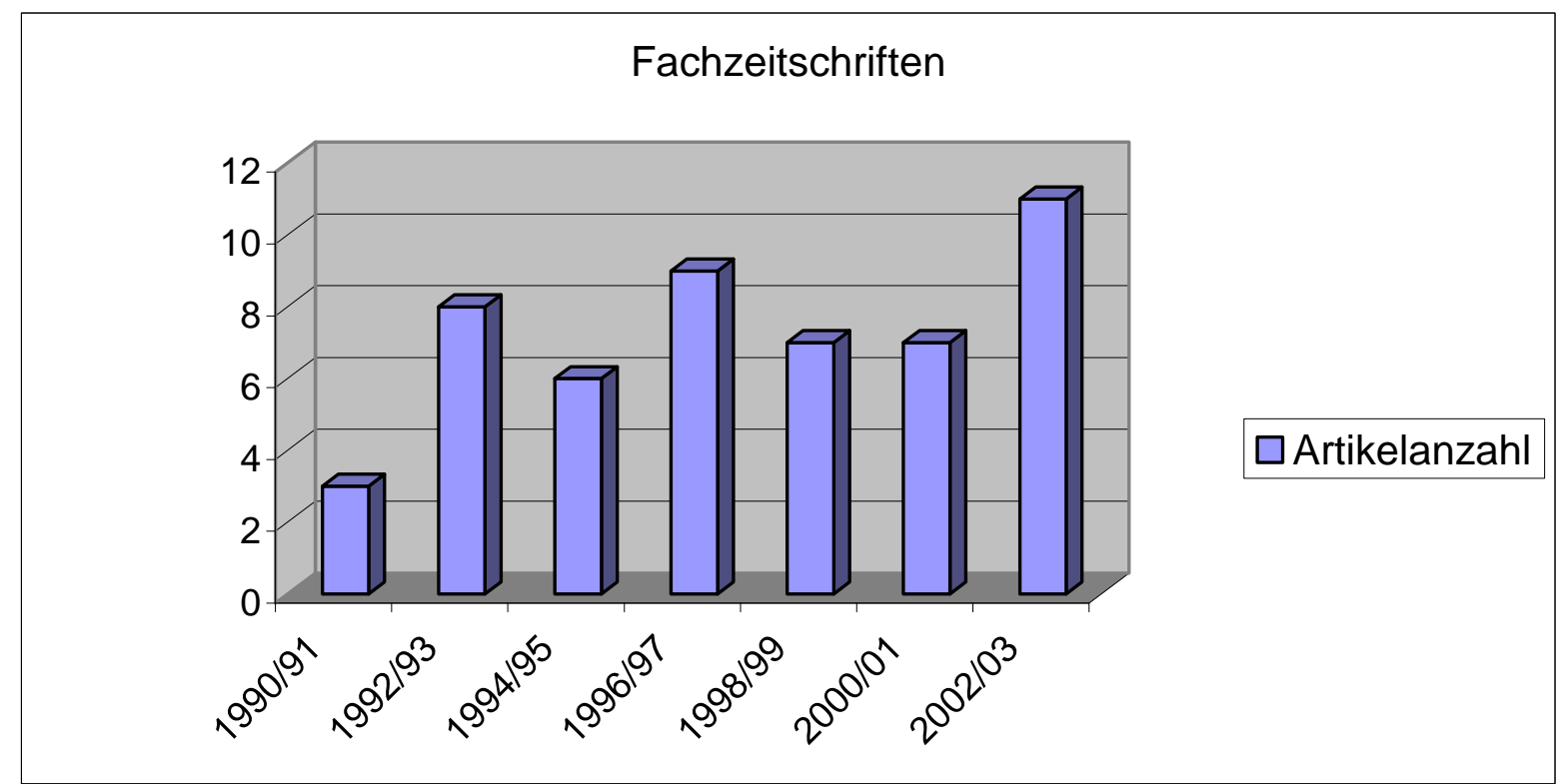

Abb. 7: Entwicklung der Artikelanzahl in medizinischen Fachzeitschriften (MMW, Internist, ZFA, Phlebologie, Dtsch Aerztebl, Dtsch Med Wochenschr) zum Thema tiefe Beinvenenthrombose. 
Auf Grund der eingeschränkten Anzahl an ausgewerteten Zeitungen und Zeitschriften erheben diese Daten allerdings nicht den Anspruch auf Vollständigkeit. Vielmehr sollen sie beispielhaft verdeutlichen, dass die Bevölkerung zumindest verstärkt über die tiefe Beinvenenthrombose informiert wurde.

Dem Diagramm in Kapitel 2.2.1.1 ist diese Entwicklung zu entnehmen. Die Summe an Patienten, die sich mit dem Verdacht auf eine tiefe Beinvenenthrombose in der Praxis vorstellten, hat signifikant zugenommen. Anhand der im Krankenhaus dokumentierten Daten konnte diese Veränderung erfasst werden. So stieg die Zahl der untersuchten Patienten von 1996 bis 2001 um 196 Fälle. Dies bedeutet eine Zunahme von rund $76 \%$. Es konnte festgestellt werden, dass im gleichen Zeitraum auch die Summe an diagnostizierten Thrombosen gestiegen ist. Deren Anteil im Verhältnis zu allen untersuchten Patienten verminderte sich leicht, allerdings nicht signifikant. Die angenommene Sensibilisierung der Bevölkerung hätte also demnach dazu geführt, dass vermehrt Patienten zum/r Arzt/Ärztin gegangen sind. Eine den Zusammenhang zwischen Patientenalter und dem Auftreten einer tiefen Beinvenenthrombose untersuchende Studie konnte zeigen, dass es zu einer signifikanten Zunahme an diagnostizierten tiefen Beinvenenthrombosen bei älteren Patienten in den letzten 10 Jahren gekommen ist; begründet wurde dieser Anstieg durch den früheren und intensiveren Einsatz diagnostischer Untersuchungsmethoden (Stein et al. 2004). Unter Berücksichtigung dieser Ergebnisse müsste eine relevante Anzahl an tiefen Beinvenenthrombosen folgenlos verlaufen. Es gilt also zu klären, inwieweit die Erkrankung immer mit schweren Risiken einhergeht oder inwieweit es subklinische Verläufe gibt, die ohne relevante Komplikationen verlaufen. 


\section{Zusammenfassung}

Die Erkrankung an einer tiefen Beinvenenthrombose ist potentiell mit schwerwiegenden Komplikationen, wie dem Auftreten einer Lungenembolie oder eines postthrombotischen Syndroms, verbunden. Sie sollte schnell, sicher und effektiv diagnostiziert und therapiert werden. Gerade in diesen Bereichen hat es in den vergangenen 10 bis 15 Jahren entscheidende Veränderungen gegeben. Eine Verlagerung sowohl der Diagnostik als auch der Therapie in den ambulanten Versorgungsbereich ist möglich geworden.

Die vorliegende retrospektive Arbeit untersucht exemplarisch, inwieweit diese Entwicklungen im Zeitraum 1990 bis 2001 zu Veränderungen in der tatsächlichen Versorgung geführt haben. Hierfür wurden in einem abgegrenzten regionalen Raum zwei größeren Einrichtungen (eine gefäßchirurgisch/phlebologische Praxis und ein Krankenhaus) untersucht.

In der beteiligten Praxis wurden die Doppeljahrgänge 1990/1, 1995/6 und 2000/1 retrospektiv ausgewertet. Die prospektive Erhebung umfasste den Zeitraum August 2001 bis April 2003. Im Krankenhaus konnten die Jahre 1996 und 2001 bearbeitet werden, da für diese Zeiträume eine lückenlose Dokumentationen vorlag. Insgesamt konnten 1195 Patienten mit tVT oder dem Verdacht auf eine tVT eingeschlossen werden. Um Verzerrungen zu vermeiden, wurden in beiden Institutionen nur Patienten berücksichtigt, die sich entweder von extern vorstellten oder überwiesen worden waren.

Die Entwicklung der Fallzahlen belegt eine signifikante Vervierfachung der Patienten mit tVT beziehungsweise Verdacht auf IVT in der Spezialistenpraxis. Im Krankenhaus ließ sich eine Zunahme um 56,7\% im Untersuchungszeitraum feststellen. Die Phlebographie als invasives Verfahren wurde zuletzt immer weniger angewendet. Anfang der 90er Jahre stellte sie noch ein wesentliches Standbein der Diagnostik dar. Sie wurde durch die die Patienten weniger belastende Duplex- oder Kompressionssonographie ersetzt. D-Dimere wurden erst im letzten Beobachtungsabschnitt 2000/1 vermehrt in die Diagnostik integriert. Bei den erhobenen Diagnosen fällt auf, dass im ambulanten Bereich distale Thrombosen stark dominieren und die schwereren proximalen Thrombosen sehr viel häufiger im Krankenhaus festgestellt wurden. 
Hier kann ein selektierender Einfluss durch die zuweisenden Ärzte angenommen werden.

Die Therapie betreffend imponiert eine nahezu komplette Verlagerung der Patienten der Spezialistenpraxis in den ambulanten Bereich (97\% in 2001). Im Krankenhaus ließ sich eine gleichgerichtete Verschiebung nachweisen. Der Anteil stationärer Behandlungen lag jedoch 2001 immer noch bei 40\%. Mit dieser Verlagerung geht ein zunehmender Einsatz von niedermolekularen Heparinen einher und zuletzt der völlige Verzicht auf die Lysetherapie.

In beiden Einrichtungen wiesen die Dokumentationen der Patienten wesentliche Defizite auf. So wurden vor allem die Symptome der Patienten häufig nur zum Teil beschrieben. Die Arztbriefe an die weiterbehandelnden Ärzte beinhalteten häufig keine Angaben zu so wesentlichen therapeutischen Hinweisen wie die Art und Dauer der Antikoagulation. Die Notwendigkeit einer Kompressionstherapie wurde nur selten angesprochen. Die Dokumentationsmängel in der retrospektiven Untersuchung ermöglichten keine sichere Beurteilung des Zusammenhangs von klinischen Symptomen und den Diagnosen. Daher wurde die retrospektive Untersuchung durch eine Prospektive ergänzt. Es sollte die Frage beantwortet werden, inwieweit die Klinik der Patienten einen Rückschluss auf die Diagnose erlaubt und welche Symptome zuweisende Ärzte veranlassen, eine weitere Abklärung beziehungsweise den Ausschluss einer IVT anzufordern.

Die prospektive Untersuchung in der Spezialistenpraxis erlaubte den Einschluss von 114 Patienten. Berücksichtigung fand der Zeitraum von August 2001 bis April 2003. Bei 12 Patienten wurde eine IVT nachgewiesen. Führende Beschwerden der Patienten waren Schmerzen (in 88\%) und Schwellungen (in 71\%). Bei der körperlichen Untersuchung dominierte die Umfangsdifferenz $(<1 \mathrm{~cm})$ in $63 \%$ und der Wadendruckschmerz in $40 \%$. Bei einer großen Spannweite von 1-90 Tagen betrug die Beschwerdedauer im Median 5 Tage vor der Vorstellung beim Spezialisten. Ein klassischer Auslöser (z.B. längere Reise, Trauma, Immobilisation) ließ sich bei weniger als $20 \%$ der Patienten ermitteln. Knapp die Hälfte wies Risikofaktoren für Thrombosen auf. Alle Patienten wurden neben der körperlichen Befundung initial duplexsonographisch untersucht. In 29\% der Fälle erbrachte diese Diagnostik keine sichere Aussage, so dass ergänzend Phlebographien und/oder D-Dimer-Tests vorgenom- 
men wurden. Wesentliche Differentialdiagnosen waren die Stamminsuffizienz (33\%), (pseudo-) radikuläre Beschwerden (20\%) und Phlebitiden (12\%).

Die Auswertung der Testgütekriterien der klinischen tVT-Zeichen ergab hohe Werte für die negative Prädiktion (z.B. 0,99 für den Wadendruckschmerz) bei gleichzeitig sehr niedrigen Werten für die positive Prädiktion (so z.B. 0,24 für den Wadendruckschmerz). Die statistische Analyse hatte zum Ergebnis, dass es den zuweisenden Ärzten nicht möglich war, eine tVT mit ausreichender Sicherheit ohne apparative Diagnostik auszuschließen. 


\section{Schlussfolgerungen}

Bezüglich der im Kapitel Zielsetzung formulierten Hypothesen lassen sich basierend auf den gewonnenen Erkenntnissen der vorliegenden Arbeit folgende Schlussfolgerungen machen:

1. Die Daten belegen - neben einer insgesamt gestiegenen Häufigkeit der Verdachtsfälle - eine Verlagerung der Diagnostik und Therapie in den ambulanten Bereich. Während diese Verlagerung in der untersuchten Spezialistenpraxis nahezu vollständig umgesetzt wurde, ist dies im berücksichtigten Krankenhaus nur teilweise geschehen. Dabei sollte jedoch berücksichtigt werden, dass die Patienten die sich im Krankenhaus vorstellten, häufiger proximale Thrombosen und somit ein ausgeprägteres Krankheitsbild aufwiesen.

2. Gerade die Daten aus der prospektiven Erhebung unterstreichen, dass es nach wie vor nicht möglich ist, eine sichere Ausschlussdiagnose der Thrombose nur basierend auf der Symptomatik zu stellen. Der Einsatz apparativer diagnostischer Verfahren ist zur sicheren Befundung nötig. Inwieweit die initiale Bestimmung der D-Dimere im primärärztlichen Bereich eine Ausschlussdiagnostik beeinflussen kann, ist anhand der aktuellen Literatur nicht abschlieBend beurteilbar. Zur Vermeidung unnötiger invasiver Diagnostik und der damit verbundenen Ressourceneinsparung sollten diesbezüglich weitere Untersuchungen durchgeführt werden.

3. Die Ergebnisse der vorliegenden Arbeit konnten den gewachsenen Stellenwert der Farbduplexsonographie gegenüber der aszendierenden Phlebographie bestätigen. Sie gilt als das Verfahren der Wahl bei Verdacht auf eine tiefe Beinvenenthrombose. Neben der hohen Spezifität und Sensitivität sind die mit dieser Untersuchungsmethode einhergehenden Vorteile hierfür ursächlich. Sie ist schnell, sicher und kostengünstig durchführbar und mit keinerlei Komplikationen verbunden. 
4. Die Messung der Fibrinogenspaltprodukte hat über den Untersuchungszeitraum zugenommen. Strittig ist jedoch nach wie vor der Stellenwert dieses Verfahrens in der Diagnostik der tiefen Beinvenenthrombose. Die Gütekriterien (niedrige Spezifität, hohe Sensitivität) erlauben lediglich in Kombinationen mit den Ergebnissen anderer Verfahren eine sichere Diagnostik. 


\section{Literaturverzeichnis}

Alonso MJL, Abinzano GML, Solano RM, Alvarez FMT, Gutierrez DJ, Munuera GL (2005): Low-molecular-weight heparin for the treatment of acute pulmonary thromboembolism. Comparison with unfractionated intravenous heparin. Ann Intern Med 224: $177-81$

Aschwanden M, Labs KH, Jeanneret C, Gehrig A, Jaeger KA (1999): The value of DDimer testing combined with structered clinical evaluation for the diagnosis of deep vein thrombosis. J Vasc Surg 30: 929-35

Aschwanden M, Labs KH, Engel H, Schwob A, Jeanneret C, Mueller-Brand J (2001): Acute deep vein thrombosis: early mobilization does not increase the frequency of pulmonary embolism. J Thromb Haemost 85: 42-6

Baarslag HJ, van Beek EJ, Koopman MM, Reekers JA (2002): Prospective study of colour duplex ultrasonography compared with contrast venography in patients suspected of having deep venous thrombosis of the upper extremities. Ann Intern Med 136: 865-72

Beighton PH (1968): Cardiovascular disease in air-travellers. Br Heart J 367-9

Bernardi E, Prandoni P, Lensing AW (1998): D-dimer testing as an adjunct to ultrasonography in patients with clinically suspected deep vein thrombosis: prospective cohort study. BMJ 317: 1037-40

Bjorgell O, Nilsson PE, Jarenros H, Nylander G (2000): Scoring of extensive deep leg vein thrombosis displayed as widespread nonfilling of contrast on phlebography. A comparison with colour Doppler ultrasonography. Acta Radiol 41: 605-11

Blättler W (1991): Ambulatory care for ambulant patients with deep venous thrombosis. J Malad Vasc (Paris) 16: 137-41

Blättler W, Partsch H, Hertel T (1998): Leitlinien zur Diagnostik und Therapie der tiefen Bein-/Beckenvenenthrombose. Phlebologie 27: 84-8

Blättler W, Gerlach HE, Partsch H: Diagnostik und Therapie der tiefen Bein- und Beckenvenenthrombose. Leitlinie der Deutschen Gesellschaft für Phlebologie. DGP, Frankfurt/Main 2003 
Boccalon H, Elias A, Chalé JJ, Cadène A, Gabriel S (2000), for the Vascular MidiPyreness Network Group. Clinical outcome and cost of hospital vs. home treatment with low-molecular-weight heparin. Arch Intern Med 160: 1769-73

Botella GF, Gomez LM (2004): New criteria for the diagnostic and treatment of deep vein thrombosis of the lower limbs. Ann Intern Med 21: 400-7

Bradley M, Bladon J, Barker H (2000): D-dimer assay for deep vein thrombosis: its role with colour Doppler sonography. Clin Radiol 55: 525-7

Brandjes DP, Büller HR, Heijboer H (1997): Randomised trial of effect of compression stockings in patients with symptomatic proximal-vein thrombosis. Lancet $\underline{349}$ : 759-62

Cogo A, Lensing AW, Koopman MM, Piovella F, Siragusa S, Wells PS (1998): Compression ultrasonography for diagnostic management of patients with clinically suspected deep vein thrombosis. BMJ $\underline{316: 17-20}$

Crippa L, D’Angelo SV, Tomassini L, Rizzi B, D’Alessandro G, D’Angelo A (1997): The utility and cost-effectivness of D-dimer measurements in the diagnosis of deep vein thrombosis. Haematologica 82: 446-51

De Moerloose P (2000): D-dimer assays for the exclusion of venous thromboembolism: which test for which diagnostic strategy? J Thromb Haemost 83: 180-1

Dempfle CE, Hafner G, Lestin HG, Töpfer G (1996): Multizentrische Evaluierung von Tina-quant D-Dimer. Lab Med 2: 31-7

Diehm C, Stammler F, Amendt K (1997): Die tiefe Venenthrombose. Dtsch Ärztebl 94: 253-63

Diehm C, Stammler F, Lawall H (2002): Akuttherapie in Zukunft ambulant? MMWFortschr Med 35: 32-8

Dietrich CF, Bauersachs RM (2002): Sonographische Thrombosediagnostik. Dtsch Med Wochenschr 127: 567-72

Dolovich LR, Ginsberg JS, Douketis JD, Holbrook AM, Cheah G (2000): A metaanalysis comparing low-molecular-weight heparins with unfractionated heparin in the treatment of venous thromboembolism: examining some unanswered questions regarding location of treatment, product type, and dosing frequency. Arch Intern Med 160: $181-8$ 
Ebell MH (2001): Evaluation of the patient with suspected deep vein thrombosis. J Fam Pract 50: 167-71

Fancher TL, White RH, Kravitz RL (2004): Combined use of rapid D-dimer testing and estimation of clinical probability in the diagnosis of deep vein thrombosis: systematic review. BMJ 329: 1236-7

Fareed J, Walenga JM, Hoppensteadt D, Huan X, Racanelli A (1988): Comparative study on the in vitro and in vivo activities of seven low-molecular-weight heparins. J Thromb Haemost 18: 3-15

Fischer T (2004): Diagnostik der tiefen Beinvenenthrombose aus hausärztlicher Sicht. Z Allgemeinmed 80: 243-54

Fischer T, Hähnel A, Schlehahn F, Bauer V, Dresler C (2004): Verdacht auf tiefe Beinvenenthrombose. Phlebologie 33: 47-52

Ginsberg JS, Kearon C, Douketis J, Turpie AGG, Brill-Edwards P, Stevens P, Panju A, Patel A, Crowther M, Andrew M (1997): The use of D-dimer testing and impedance plethysmographic examination in patients with clinical indications of deep vein thrombosis. Arch Intern Med 157: 1077-81

Greinacher A (1997): Neue antithrombotische Medikamente. Internist 38: 680-7

Grohmann G (1999): Physikalische Methoden zur Prophylaxe von Venenthrombosen. Ärztebl Thüringen 10: 666-8

Hausman W (1999): Risikoadaptierte Thromboseprophylaxe. PZ Prisma 6: 73-8

Heit JA, Silverstein MD, Mohr DN (2001): The epidemiology of venous thromboembolism in the community. J Thromb Haemost 86: 452-63

Herrmann K, Waggershausen T, Bonel H, Glaser C, Sittek H, Reiser M (1998): Kontrastmitteluntersuchungen des Venensystems. Radiologe 38: 570-7

Hirsh J, Warkentin TE, Raschke R, Granger C, Ohman EM, Dalen JE (1998): Heparin and low-molecular-weight heparin. Mechanisms of action, pharmacokinetics, dosing, considerations, monitoring, efficacy and safety. Chest 114: 489-510

Holmstrom M, Lindmarker P, Granquist S, Johnsson H, Lockner D (1997): A 6-month venographic follow-up in 164 patients with acute deep vein thrombosis. J Thromb Haemost 78: 803-7 
Hyers TM, Agnelli G, Hull RD, Morris TA, Samama M, Tapson V (2001): Antithrombotic therapy for venous thromboembolic disease. Chest 119: 176-93

Ilkhanipour K, Wolfson AB, Walker H, Cillo J, Rolniak S, Cockley P, Mooradian D, Kaplan S (2004): Combining clinical risk with D-dimer testing to rule out deep vein thrombosis. J Emerg Med 27: 233-9

Kearon C (2001): Epidemiology of venous thromboembolism. Semin Vasc Med 1: 726

Kearon C, Julian JA, Newman TE, Ginsberg JS (1998): Noninvasive diagnosis of deep venous thrombosis. Ann Intern Med 128: 663-77

Kearon C, Ginsberg JS, Douketis J (2001): Managment of suspected deep venous thrombosis in outpatients by using clinical assessment and D-dimer testing. Ann Intern Med 135: 108-11

Kelly J, Rudd A, Lewis RR, Hunt BJ (2002): Plasma D-dimers in the diagnosis of venous thromboembolism. Arch Intern Med 162: 747-56

Knecht MF (1994): Das Fibrinspaltprodukt D-dimer. Klinische Anwendung in Diagnostik und Therapiekontrolle. Med Welt 45: 140-6

Knecht MF, Heinrich F (1995): Rapid D-dimer Assay in deep venous thrombosis and pulmonary embolism. J Thromb Haemost 73: 899-1534

Knecht MF, Heinrich F (1997): Clinical evaluation of an immunturbidimetric D-Dimer assay in the diagnostic procedure of deep vein thrombosis and pulmonary embolism. Thromb Res 88: 413-7

Koopman MM, Prandoni P, Piovella F, Ockelford PA, Brandjes DP, van der Meer J, Gallus G, Simonneau G, Chesterman CH, Prins MH (1996): Treatment of venous thrombosis with intravenous unfractioned heparin administered in the hospital compared with subcutaneous low-molecular-weight heparin administered at home. $\mathrm{N}$ Engl J Med 334: 682-7

Kozman H, Flemmer MC, Rahnama M (1997): Deep venous thrombosis: prediction by D-dimer? South Med J 90: 907-10

Kraaijenhagen RA, Lensing AWA, Lijmer GJ, Prandoni P, Prins MH, Ginsberg JS, Buller HR (1997): Diagnostic strategies for the management of patients with clinically suspected deep vein thrombosis. Curr Opin Pulm Med $\underline{3}$ : 268-74 
Le Gal G, Righini M, Roy PM, Sanchez O, Aujesky D, Perrier A, Bounameaux H (2006): Value of D-dimer testing for the exclusion of pulmonary embolism in patients with previous venous thromboembolism. Arch Intern Med 166: 176-80

Legnani C, Pancani C, Palareti G, Guazzaloca G, Coccheri C (1999): Contribution of a new, rapid, quantitative and automated method for D-dimer measurement to exclude deep vein thrombosis in symptomatic outpatients. Blood Coagul Fibrinolysis 10: $69-74$

Levine M, Gent M, Hirsh J, Leclerc J, Anderson D, Weitz J, Ginsberg J, Turpie AG, Demers C, Kovacs M (1996): A comparison of low-molecular-weight heparin administered primarily at home with unfractionated heparin administered in the hospital for proximal deep-vein thrombosis. N Engl J Med 334: 677-81

Martinelli I, Mannucci PM, De Stefano U, Taioli E, Rossi V, Crosti F, Paciaroni K, Leone G, Faioni EM (1998): Different risk of thrombosis in four coagulation defects associated with inherited thrombophilia: a study of 150 families. Blood 92: 2353-8

Mateo J, Oliver A, Borrell M, Sala N, Fontcuberta J, and the EMET Group (1997): Laboratory evaluation and the clinical characteristics of 2132 consecutive unselected patients with venous thromboembolism. J Thromb Haemost 77: 444-51

Merli G, Spiro TE, Olsson CG, Abildgaard U, Davidson B, Eldor A, Elias D, Grigg A, Musset D, Rodgers GM (2001): Subcutaneous enoxaparin twice daily compared with intravenous unfractionated heparin for treatment of venous thrombembolic disease. Ann Intern Med 134: 191-202

Miller N, Satin R, Tousignant L, Sheiner NM (1996): A prospective study comparing duplex scan and venography for diagnosis of lower extremity deep vein thrombosis. Cardiovasc Surg 4: 505-8

Müller-Lisse UL, Müller-Lisse GU, Holzknecht N, Reiser M (1998): Ultraschalluntersuchungen der Venen. Radiologe 38: 560-9

Noren A, Ottosson E, Sjunnesson M (2002): A detailed analysis of equivocal duplex findings in patients with suspected deep venous thrombosis. J Ultrasound Med 21: 1375-83

O`Brien B, Levine M, Willan A, Goeree R, Haley S, Blackhouse G (1999): Economic evaluation of outpatient treatment with low-molecular-weight-heparin for proximal vein thrombosis. Arch Intern Med 159: 2298-304 
Offermann M, Kopechen J, Graulich B (2001): Die ambulante Therapie der akuten tiefen Beinvenenthrombose. Erfahrungen aus zwei Praxen für Gefäßkrankheiten in Deutschland. Dtsch Med Wochenschr 126: 1164-7

Oudega R, Hoes AW, Moons K (2005 a): The Wells Rule does not adequately rule out deep venous thrombosis in primary care patients. Ann Intern Med 143: 140-2

Oudega R, Moons K, Hoes A (2005 b): Limited value of patient history and physical examination in diagnosing deep vein thrombosis in primary care. J Fam Pract 10: 8691

Oudega R, Moons K, Hoes A (2005 c): Ruling out deep venous thrombosis in primary care. A simple diagnostic algorithm including D-dimer testing. J Thromb Haemost 94: 200-5

Partsch H (2001): Therapy of deep vein thrombosis with low molecular weight heparin, leg compression and immediate ambulation. Vasa 30: 195-204

Partsch H, Blättler W (2000): Compression and walking versus bed rest in the treatment of proximal deep venous thrombosis with low molecular weight heparin. $J$ Vasc Surg 32: 861-9

Perone N, Bounameaux H, Perrier A (2001): Comparison of four strategies for diagnosing deep vein thrombosis: a cost-effectivness analysis. Am J Med 110(1): 69-70

Perrier A, Bounameaux H (2001): Cost-effective diagnosis of deep vein thrombosis and pulmonary embolism. J Thromb Haemost 86: 475-87

Perrier A, Desmarais S, Miron MJ (1999): Non-invasive diagnosis of venous thromboembolism in outpatients. Lancet 353: 190-5

Prandoni P, Carnovali M, Marchiori A (2004): Subcutaneous adjusted-dose unfractionated heparin vs fixed-dose low-molecular-weight heparin in the initial treatment of venous thromboembolism. Arch Intern Med 164: 1077-83

Rathbun SW, Whitsett TL, Raskob GE (2004): Negative D-dimer result exclude recurrent deep venous thrombosis. Ann Intern Med 141: 839-45

Rudofsky G, Michler E (2002): Bildgebende Diagnostik der tiefen Bein- und Beckenvenenthrombose. Internist 43: 27-35

SAS Institute Inc. SAS/STAT: User's Guide Version 8, SAS, Cary 1999 
Schellong SM, Schwarz T (2002): Differenzialtherapie der tiefen Beinvenenthrombose. Internist 43: 36-46

Schellong SM, Schwarz T, Kroop J (1999): Bed rest in deep vein thrombosis and the incidence of pulmonary embolism. J Thromb Haemost 82: 127-9

Schraibman IG, Milne AA, Royle EM: Home versus in-patient treatment for deep vein thrombosis (Cochrane-Review). In: The Cochrane Library, Issue 1, John Wiley \& Sons, Ltd., Oxford/UK 2004

Schröder F, Weiss T (2002): Niedermolekulare Heparine. Dtsch Med Wochenschr 127: $1645-7$

Schwarz T, Schmidt B, Höhlein U, Beyer J, Schröder HE, Schellong SM (2001): Eligibility for home treatment of deep vein thrombosis: prospective study. BMJ $\underline{322}$ : 1212-3

Seligsohn U, Lubetsky A (2001): Genetic susceptibility to venous thrombosis. N Engl J Med 344: 1222-31

Silverstein MD, Heit JA, Mohr DN (1998): Trends in the incidence of deep vein thrombosis and pulmonary embolism: a 25-year population based study. Arch Intern Med 158: 585-93

Spandorfer JM, Lynch S, Weitz HH, Fertel S, Merli GJ (1999): Use of enoxaparin for the chronically anticoagulated patient before and after procedures. Am J Cardiol $\underline{84}$ : 478-80

Spengel FA, von Liebe S (1998): Venöse Thrombosen - eine diagnostische und therapeutische Herausforderung. Radiologe 38: 549-53

Stammler F, Diehm C (1998): Niedermolekulare Heparine bei venöser Thromboembolie. Dtsch Med Wochenschr 123: 604-11

Stein PD, Hull RD, Kayali F, Ghali WA, Alshab AK, Olson RE (2004): Venous thromboembolism according to age. Arch Intern Med 164: 2260-5

Tatò F (2002): Diagnostische Strategien für die venöse Thromboembolie. Phlebologie 31 : $150-5$

Tovey C, Wyatt S (2003): Diagnosis, investigation, and management of deep vein thrombosis. BMJ 326: 1180-4 
Van Boven HH, Vandenbroucke JP, Briet E, Rosendaal FR (1999): Gene-gene and gene-environment interactions determine risk of thrombosis in families with inherited antithrombin deficiency. Blood 94: 2590-4

Van den Belt AG, Prins MH, Lensing AW, Castro AA, Clark OA, Atallah AN: Fixed dose subcutaneous low molecular weight heparins versus adjusted dose unfractionated heparin for venous thromboembolism (Cochrane Review). In: The Cochrane Library, Issue 1, John Wiley \& Sons, Ltd., Oxford/UK 2004

Van Dongen CJ, Mac Gillavry M, Prins M: Once versus twice daily LWMH for the initial treatment of venous thromboembolism. In: The Cochrane Library, Issue 3, John Wiley \& Sons, Ltd., Oxford/UK 2005

Warkentin TE (2002): Platelet count monitoring and laboratory testing for heparininduced thrombozytopenia. Arch Pathol Lab Med 126: 1415-23

Warkentin TE, Greinacher A (2004): Heparin-induced thrombocytopenia: recognition, treatment, and prevention: the Seventh ACCP Conference on Antithrombotic and Thrombolytic Therapy. Chest 126: 311-27

Warkentin TE, Levine MN, Jirsh J, Horsewood P, Roberts RS, Tech M, Gent M, Kelton JG (1995): Heparin-induced thrombocytopenia in patients treated with low molecular weight heparin or unfractionated heparin. N Engl J Med 332: 1330-5

Weber (1998): Qualitätssicherung bei der Extremitätenphlebographie. Radiologe $\underline{38}$ $578-85$

Weitz JI (1997): Low molecular weight heparins. N Engl J Med 337: 688-98

Wells PS, Anderson DR, Bormanis J, Guy F, Mitchell M, Gray L, Clement C, Robinson KS, Lewandowski B (1997): Value of assessment of pretest probability of deep vein thrombosis in clinical management. Lancet 350: 1795-8

Wells PS, Kovacs MJ, Bormanis J, Forgie MA, Goudie D, Morrow B (1998): Expanding eligibility for outpatient treatment of deep venous thrombosis and pulmonary embolism with low-molecular heparin: a comparison of patient self-injection with homecare injection. Arch Intern Med 158: 1809-12

Wells PS, Anderson DR, Ginsberg J (2000): Assessment of deep vein thrombosis or pulmonary embolism by the combined use of clinical model and noninvasive tests. $J$ Thromb Haemost 26: 643-56 
Wells PS, Anderson DR, Rodger M (2003): Evaluation of D-dimer in the diagnosis of suspected deep-vein thrombosis. N Engl J Med 349: 1227-35

Wheeler HB, Anderson FA (1995): Diagnostic methods for deep vein thrombosis. J Thromb Haemost 25: 6-26 


\section{Anhang}

\subsection{Retrospektiver Erfassungsbogen}

Pat.nr.

Aufnahme:

Überweisung: von wem:

Text:
Geb.datum:

Entlassung:

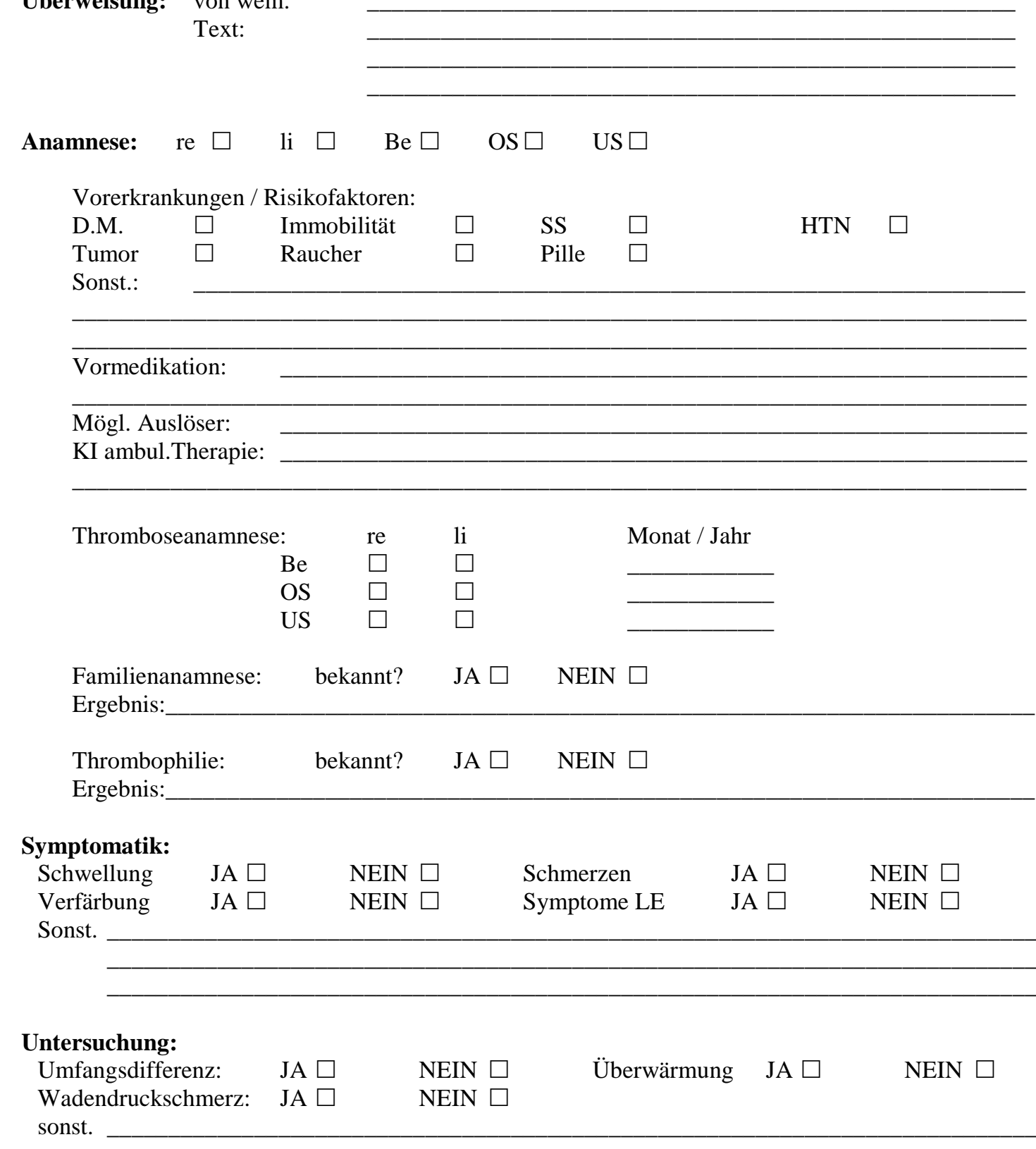

\section{Diagnostik:}

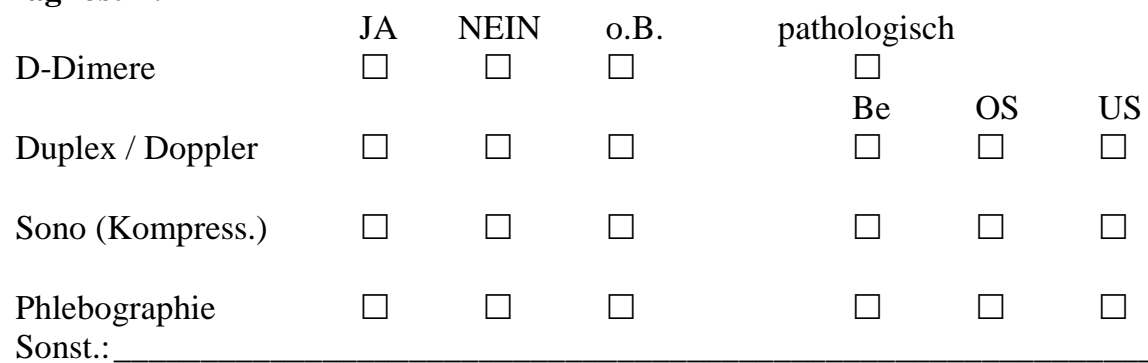




\section{Diagnose:}

\begin{tabular}{lllll} 
Thrombose & Be $\square \quad$ OS $\square \quad$ US $\square$ & Ausschluss Thrombose $\square$ \\
V.a. Thrombose & Be $\square \quad$ OS $\square \quad$ US $\square$ \\
LE $\square$ & Ausschluss LE $\square$ & & \\
\hline
\end{tabular}

Sonst.:

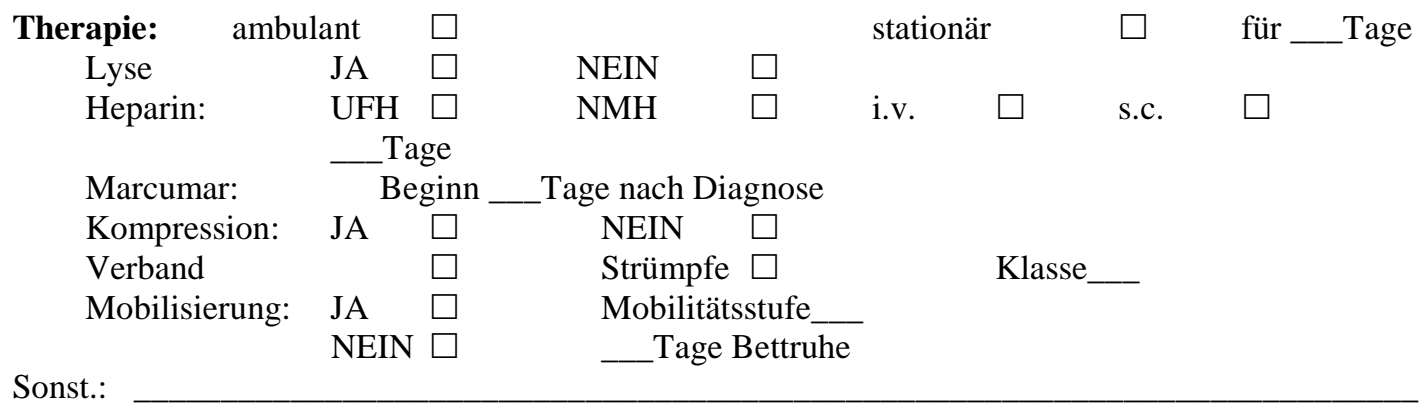

\section{Entlassungsbrief:}

JA

NEIN

Tage nach Entlassung erstellt

Marcumar erwähnt?

JA

NEIN

Dauer:

INR:

Quick:

Heparin erwähnt?

$$
\begin{aligned}
& \text { UFH } \\
& \text { Dauer: }
\end{aligned}
$$

JA $\square$

NEIN

$\mathrm{NMH}$

Kompression erwähnt?

JA

NEIN

Dauer:

\begin{tabular}{|c|c|c|c|}
\hline $\begin{array}{l}\text { Tumordiagnostik durchgeführt? } \\
\text { Ergebnis: }\end{array}$ & $\mathrm{JA}$ & $\square$ & NEIN \\
\hline empfohlen? & JA & $\square$ & NEIN \\
\hline \multirow{2}{*}{$\begin{array}{l}\text { Thrombophiliediagnostik durchgeführt? } \\
\text { Ergebnis: }\end{array}$} & JA & $\square$ & NEIN \\
\hline & JA & $\square$ & NEIN \\
\hline
\end{tabular}

Klasse:

Sonst.

Verlaufskontrollen:

$\begin{array}{lllll}\text { Angaben vorhanden? } & \text { JA } & \text { NEIN } & \text { pos. } & \text { neg. } \\ \text { Blutungen } & \square & \square & \square & \square \\ \text { Rezidiv } & \square & \square & \square & \square \\ \text { LE } & \square & \square & \square & \square \\ \text { PTS } & \square & \square & \square & \square\end{array}$




\title{
8.2 Prospektiver Erfassungsbogen
}

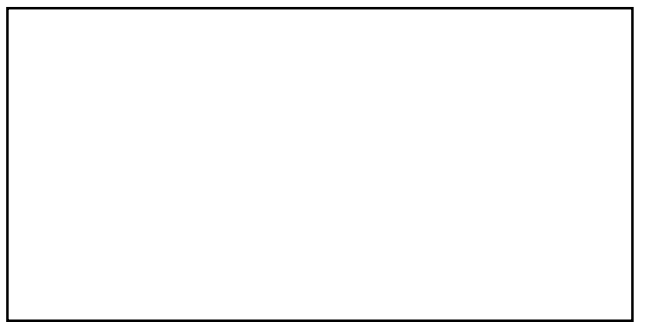

\author{
Chirurgische \\ Gemeinschaftspraxis \\ Dr. med. M. Klöpper, Dr. med. V. Bauer, \\ Dr. med. Chr. Dresler, St. Breme \\ Eschenschlag 7, 37154 Northeim \\ Tel. 05551-914170, Fax: 9141710
}

\section{Untersuchungsblatt Thrombose}

Datum:

\section{Anamnese:}

Beschwerdebeginn:

Auslöser:

$\overline{\text { (Reise, OP, Immobilisation, etc.) }}$

Schwellung:

$\square$ ja $\square$ nein

Schmerzen:

$\square$ ja

nein

sonstige Beschwerden:

Thromboseanamnese:
$\mathrm{Re} \quad \mathrm{Li}$
$\square \quad \square$ US
$\square \quad \square$ OS
$\square$ Becken
$\square \quad \square$ sonstige,

Bisherige Maßnahmen (Hausarzt):

Kompressionsverband: $\square$ ja $\square$ nein Kp.strumpf vorhanden: $\square$ ja $\square$ nein
Familiäre Vorbelastung: $\quad \square$ nein

$\square$ ja,

Thrombophiliediagnostik: $\square$ nein

$\square$ ja，

Überweisung: $\square$ ja $\quad \square$ nein

Antikoagulation: $\square$ ja $\square$ nein (welche:)

Klinischer Befund:

\begin{tabular}{rl|l}
\multicolumn{1}{c}{ Re. } & \multicolumn{2}{c}{ Li. } \\
\cline { 2 - 3 } Umfang (cm): Knöchel
\end{tabular}

Verfärbung $\square$ ja $\quad \square$ nein

Homann: $\square$ pos. $\square$ neg

(Dorsalextension Fuß)

Wadendruckschmerz:

Duplexsonographie:

V. fem.

o.B.

pathologisch,

V. pop.

o.B

pathologisch,

Muskelvenen:
o.B.
pathologisch,

Diagnose: 


\section{Danksagung}

Ich danke meinem Betreuer Dr. Thomas Fischer. Neben zahlreichen Anregungen hat er mich stets bei auftretenden Problemstellungen unterstützt.

Ebenso danke ich Herrn Prof. Dr. med. Michael M. Kochen, MPH, FRCGP für die freundliche Überlassung des Themas, die konstruktive Kritik und die gewährte Unterstützung.

Mein besonderer Dank gilt meinem Vater, der sich sehr intensiv um die äußere Form der Arbeit gekümmert hat. 


\section{Lebenslauf}

Ich wurde am 24.06.77 als Sohn von Frau Dr. Ortrun Schlehahn und Dipl.-Math. Gunter Schlehahn in Leipzig geboren. Meinen Hochschulabschluss absolvierte ich am Gymnasium Ernestinum in Celle 1997. Im direkten Anschluss leistete ich meinen Grundwehrdienst ab.

Zum Wintersemester 1998/1999 nahm ich mein Studium der Humanmedizin an der Universität zu Köln auf. Zum Sommersemester 1999 wechselte ich an die Universität Göttingen. Meinen vorklinischen Abschnitt konnte ich im März 2001 abschließen. Im Frühjahr 2002 folgte das erste Staatsexamen, zwei Jahre später das zweite Staatsexamen. Mein praktisches Jahr, das ich in den Fächern Innere Medizin, Chirurgie und Anästhesie ableistete, konnte ich mit dem dritten Staatsexamen und der Gesamtnote 2 im Oktober 2005 beenden.

Famulaturen nahm ich in mehreren Krankenhäusern und Facharztpraxen in den Fachdisziplinen Chirurgie, Innere Medizin, Urologie, Hals-Nasen-Ohrenheilkunde sowie Dermatologie war.

Im November 2005 konnte ich aufgrund langjähriger studentischer Mitarbeit eine Stelle als Giftberater im Giftinformationszentrum-Nord antreten. Im Mai 2006 wechselte ich an das Albert-Schweitzer-Krankenhaus Northeim, wo ich seitdem in der internistischen Abteilung des Hauses als Assistenzarzt tätig bin. 